\title{
Effects of dust charge variation on electrostatic waves in dusty plasmas with temperature anisotropy
}

\author{
M.C. de Juli \\ Centro de Rádio-Astronomia e Astrofísica Mackenzie - CRAAM, Universidade Presbiteriana Mackenzie, \\ Rua da Consolação 896, CEP: 01302-907, São Paulo, SP, Brasil \\ R.S. Schneider ${ }^{\dagger}$ and L. F. Ziebell ${ }^{1}$ \\ Instituto de Física, Universidade Federal do Rio Grande do Sul, \\ Caixa Postal 15051, CEP: 91501-970, Porto Alegre, RS, Brasil. \\ R. Gaelzer \\ Instituto de Física e Matemática, Universidade Federal de Pelotas, \\ Caixa Postal 354 - Campus UFPel, CEP: 96010-900, Pelotas, RS, Brasil
}

(Received on 14 January, 2009)

\begin{abstract}
We utilize a kinetic approach to the problem of wave propagation in dusty plasmas, taking into account the variation of the charge of the dust particles due to inelastic collisions with electrons and ions. The components of the dielectric tensor are written in terms of a finite and an infinite series, containing all effects of harmonics and Larmor radius. The formulation is quite general and valid for the whole range of frequencies above the plasma frequency of the dust particles, which are assumed motionless. The formulation is employed to the study of electrostatic waves propagating along the direction of the ambient magnetic field, in the case for which ions and electrons are described by bi-Maxwellian distributions. The results obtained in a numerical analysis corroborate previous analysis, about the important role played by the dust charge variation, particularly on the imaginary part of the dispersion relation, and about the very minor role played in the case of electrostatic waves by some additional terms appearing in the components of the dielectric tensor, which are entirely due to the occurrence of the dust charge variation.
\end{abstract}

Keywords: Electrostatic waves; Kinetic theory; Magnetized dusty plasmas; Dust charge fluctuation; Wave propagation

\section{INTRODUCTION}

In the development of a proper kinetic formulation for the analysis of wave propagation and damping in a plasma containing a population of charged dust particles, it is necessary to take into account the process of charging of the dust grains. However, despite the recognized importance of this effect to the propagation and damping of waves $[1,2]$, and despite the recognized need of a kinetic formulation including effects due to the dust charging for proper evaluation of the wave damping $[3,4]$, most of the published literature utilizes fluid theory to describe the dusty plasmas, and only a small fraction of the published papers take into account the collisional charging of the dust particles [5-8].

Motivated by the importance of the use of a proper kinetic formulation for the analysis of wave behavior in dusty plasmas with dust grains of variable charge, in a recent paper we have developed a very general mathematical formulation, writing the expressions for the components of the dielectric tensor in terms of an infinite and a finite summation, formally incorporating effects of all cyclotron harmonics and all orders of Larmor radius, keeping effects due to the dust charge variation [9]. The formulation developed is very general in terms of frequency range and direction of propagation, and it is expected to be very useful for application to the study of wave propagation in dusty plasmas in a variety of situations. As an example of application, in Ref. [9] we have also included a brief discussion of the particular case of electrostatic waves

\footnotetext{
${ }^{\dagger}$ In Memoriam

*Electronic address: ziebell@if.ufrgs.br
}

propagating along the direction of the ambient magnetic field, assuming the case of Maxwellian distributions for electrons and ions in the equilibrium, including some results originated from numerical solutions of the dispersion relation.

In the present paper, we resume the use of the formulation developed and presented in Ref. [9], in order to investigate the dispersion relation for electrostatic waves in a dusty plasma, considering the case of bi-Maxwellian distribution functions for ions and electrons. The analysis therefore includes simultaneously the effects of the presence of dust particles, including the effect of the dust charge variation, and the effect of the anisotropy in electron and ion temperatures.

The structure of the paper is the following. In Section 2 we briefly outline the model used to describe the dusty plasma and present essential features of the kinetic formulation which leads to the components of the dielectric tensor which are necessary for the dispersion relation. We also present a discussion of the dispersion relation of the electrostatic waves, emphasizing the particular case of waves propagating along the direction of the ambient magnetic field, in the case of bi-Maxwellian distributions for the electrons and ions in the equilibrium. In Section 3 some results obtained from numerical solution of the dispersion relation are presented and discussed. The conclusions are presented in Section 4. Appendix A shows details of the evaluation of the basic integrals appearing in the components of the dielectric tensor, for the case of bi-Maxwellian distributions for electrons and ions. Appendix B presents some details on the evaluation of the average frequency of inelastic collisions between electrons and ions and dust particles, and Appendix $\mathrm{C}$ briefly discusses some features related to the equilibrium condition of the process of collisional charging of the dust particles. 


\section{THE HOMOGENEOUS MAGNETIZED DUSTY PLASMA MODEL AND THE COMPONENTS OF THE DIELECTRIC TENSOR}

The present paper is an application of the general formalism recently appeared in Ref. [9]. Since the formalism is easily available, the whole set of necessary expressions and definitions will not be repeated here, for the sake of economy of space. Nevertheless, it may be useful to repeat here a short account of basic features, which will be made in the following paragraphs.

In our general kinetic formulation we consider a plasma in a homogeneous ambient magnetic field $\mathbf{B}_{\mathbf{0}}=B_{0} \mathbf{e}_{\mathbf{z}}$, in the presence of spherical dust grains with constant radius $a$ and variable electric charge $q_{d}$. We assume that the electrostatic energy of the dust particles is much smaller than their kinetic energy, the so-called weakly coupled dusty magneto-plasmas. This condition is not very restrictive, since a large variety of natural and laboratory dusty plasmas can be classified as weakly coupled [10]. The charging of the dust grains is assumed to occur by the capture of plasma electrons and ions during inelastic collisions between these particles and the dust particles. Since the electron thermal speed is much larger than the ion thermal speed, the equilibrium dust charge becomes preferentially negative. The cross-section for the charging process of the dust particles is modeled by we expressions derived from the OML theory (orbital motion limited theory) [11, 12].

Although we assume the occurrence of a magnetic field, the model which we use for the dust charging does not take into account the effect of the magnetic field, being valid only for parameters such that the size of the dust particles is much smaller than the electron Larmor radius. This feature is important, since it has been shown that the effect of the magnetic field on the charging of the dust particles can be safely neglected when the size of the dust particles is much smaller than the electron Larmor radius $[13,14]$.

Moreover, dust particles are assumed to be immobile, and consequently the validity of the proposed model will be restricted to waves with frequency much higher than the characteristic dust frequencies. In particular we consider the regime in which $\left|\Omega_{d}\right| \ll \omega_{p d}<\omega$, where $\omega_{p d}$ and $\Omega_{d}$ are the plasma frequency and the cyclotron frequency of the dust particles, respectively. This condition therefore excludes the analysis of the modes which can arise from the dust dynamics, as the socalled dust-acoustic wave.

Using this basic framework, we arrive to expressions for the components of the dielectric tensor which can be separated into two kinds of contributions $[15,16]$

$$
\varepsilon_{i j}=\varepsilon_{i j}^{C}+\varepsilon_{i j}^{N}
$$

Repeating here the commentary which has already appeared along with previous presentations of the formalism, the term $\varepsilon_{i j}^{C}$ is formally identical, except for the $i z$ components, to the dielectric tensor of a magnetized homogeneous conventional plasma of electrons and ions, with the resonant denominator modified by the addition of a purely imaginary term which contains the inelastic collision frequency of dust particles with electrons and ions. For the $i z$ components of the dielectric tensor, in addition to the term obtained with the prescription above, there is a term which is proportional to this inelastic collision frequency. The term $\varepsilon_{i j}^{N}$ arises only due to the process of variation of the charge of the dust particles, and vanishes in the case of a dustless plasma. Although the formal contribution due to this kind of term is already recognized in the literature since at least the first years of the past decade, its contribution to numerical analysis of the dispersion is usually neglected. One notices that the form of the $\varepsilon_{i j}^{N}$ components is strongly dependent on the model used to describe the charging process of the dust particles.

Explicit expressions for the components $\varepsilon_{i j}^{C}$ and $\varepsilon_{i j}^{N}$ can be found in Refs. [9, 15, 16]. Particularly, in Ref. [9] the expressions appear according to the formulation and definitions to be used in the present paper. According to this novel formulation, the components of the dielectric tensor can be written in terms of a double summation, one finite and another infinite, in which the contribution of harmonics and Larmor radius terms is shown explicitly. For the 'conventional' contribution, a component $\varepsilon_{i j}^{C}$ can be written as follows

$$
\varepsilon_{i j}^{C}=\delta_{i j}+\delta_{i z} \delta_{j z} e_{z z}+N_{\perp}^{\delta_{i z}+\delta_{j z}} \chi_{i j}
$$

while a component $\varepsilon_{i j}^{N}$ is written as

$$
\varepsilon_{i j}^{N}=\mathcal{U}_{i} S_{j}
$$

In the case of electrostatic waves (ES waves) and parallel propagation, which the subject of the present application, the dispersion relation is simply given by $\varepsilon_{z z}=0$. Therefore, we present here only the explicit expressions for the $z z$ contributions to the dielectric tensor. For general distributions and arbitrary directions of propagation, the contribution to the 'conventional' part appears as follows

$$
\begin{gathered}
\chi_{z z}=\frac{v_{*}^{2}}{c^{2}} \sum_{\beta} \frac{1}{r_{\beta}^{2}} \frac{\omega_{p \beta}^{2}}{\Omega_{*}^{2}} \frac{1}{n_{\beta 0}} \sum_{m=1}^{\infty}\left(\frac{q_{\perp}}{r_{\beta}}\right)^{2(m-1)} \\
\times \sum_{n=-m}^{m} a(|n|, m-|n|)\left[J\left(n, m, 2 ; f_{\beta 0}\right)+i J_{v}\left(n, m, 1 ; f_{\beta 0}\right)\right] \\
e_{z z}=-\frac{1}{z^{2}} \sum_{\beta} \frac{\omega_{p \beta}^{2}}{\Omega_{*}^{2}} \frac{1}{n_{\beta 0}} \int d^{3} u \frac{u_{\|}}{u_{\perp}} \mathcal{L}\left(f_{\beta 0}\right) \\
+\frac{1}{z^{2}} \sum_{\beta} \frac{\omega_{p \beta}^{2}}{\Omega_{*}^{2}} \frac{1}{n_{\beta 0}} a(0,0)\left[J\left(0,0,2 ; f_{\beta 0}\right)+i J_{v}\left(0,0,1 ; f_{\beta 0}\right)\right]
\end{gathered}
$$

where

$$
\begin{gathered}
J\left(n, m, h ; f_{\beta 0}\right) \equiv \int d^{3} u \frac{z u_{\|}^{h} u_{\perp}^{2(m-1)} u_{\perp} L\left(f_{\beta 0}\right)}{z-n r_{\beta}-q_{\|} u_{\|}+i \tilde{v}_{\beta d}^{0}}, \\
J_{v}\left(n, m, h ; f_{\beta 0}\right)=\int d^{3} u \frac{\tilde{v}_{\beta d}^{0} u_{\|}^{h} u_{\perp}^{2(m-1)} u_{\perp} \mathcal{L}\left(f_{\beta 0}\right)}{z-n r_{\beta}-q_{\|} u_{\|}+i \tilde{v}_{\beta d}^{0}}, \\
L=\frac{1}{\gamma}\left[\left(\gamma-\frac{q_{\|}}{z} u_{\|}\right) \frac{\partial}{\partial u_{\perp}}+\frac{q_{\|}}{z} u_{\perp} \frac{\partial}{\partial u_{\|}}\right],
\end{gathered}
$$




$$
\mathcal{L}=u_{\|} \frac{\partial}{\partial u_{\perp}}-u_{\perp} \frac{\partial}{\partial u_{\|}}
$$

with the dimensionless variables

$$
\begin{gathered}
z=\frac{\omega}{\Omega_{*}}, \quad q_{\|, \perp}=\frac{k_{\|, \perp} v_{*}}{\Omega_{*}}, \quad u_{\|, \perp}=\frac{p_{\|, \perp}}{m_{\beta} \nu_{*}}, \\
r_{\beta}=\frac{\Omega_{\beta}}{\Omega_{*}}, \quad \tilde{v}_{\beta d}^{0}=\frac{v_{\beta d}^{0}(u)}{\Omega_{*}}, \quad u=\left(u_{\|}^{2}+u_{\perp}^{2}\right)^{1 / 2}
\end{gathered}
$$

where the inelastic collision frequency between plasma particles and dust particles is given by

$$
v_{\beta d}^{0}(u)=\frac{\pi a^{2} n_{d 0} v_{*}}{u}\left(u^{2}-\frac{2 q_{d 0} q_{\beta}}{a m_{\beta} v_{*}^{2}}\right) H\left(u^{2}-\frac{2 q_{d 0} q_{\beta}}{a m_{\beta} v_{*}^{2}}\right) .
$$

The quantities $\Omega_{*}$ and $v_{*}$ are a characteristic frequency and a velocity, respectively, which are considered convenient for normalization in the case of a particular application. For the present application, we use $\Omega_{*}=\omega_{p e 0}^{0}$ and $v_{*}=c_{s}$, where $c_{s}=\left(T_{e} / m_{i}\right)^{1 / 2}$ is the ion-sound velocity and $\omega_{p e 0}^{0}$ is the equilibrium electron plasma angular frequency in the absence of dust. The quantity $q_{d 0}$ is the equilibrium value of the charge of the dust particles, which we will denote as $q_{d 0}=-Z_{d 0} e$.

The contribution of the 'new' part, for general distributions and directions of propagation, appears as follows,

$$
\begin{aligned}
& \mathcal{U}_{z}=\frac{1}{z} \frac{1}{z+i\left(\tilde{\mathrm{v}}_{c h}+\tilde{\mathrm{v}}_{1}\right)} \sum_{\beta} \frac{\omega_{p \beta}^{2}}{\Omega_{*}^{2}} \frac{1}{n_{\beta 0}} \sum_{m=0}^{\infty} \sum_{n=-m}^{+m}\left(\frac{q_{\perp}}{r_{\beta}}\right)^{2 m} \\
& \times a(|n|, m-|n|) J_{U}\left(n, m, 1,0 ; f_{\beta 0}\right), \\
& S_{z}=-\frac{a \Omega_{*}}{2 v_{*}} \frac{1}{z} \sum_{\beta} \frac{\omega_{p \beta}^{2}}{\Omega_{*}^{2}} \frac{1}{n_{\beta 0}} \sum_{m=0}^{\infty} \sum_{n=-m}^{+m}\left(\frac{q_{\perp}}{r_{\beta}}\right)^{2 m} \\
& \times a(|n|, m-|n|)\left[J_{v L}\left(n, m, 1 ; f_{\beta 0}\right)+i J_{v v}\left(n, m ; f_{\beta 0}\right)\right] \\
& +\frac{a \Omega_{*}}{2 v_{*}} \frac{1}{z} \sum_{\beta} \frac{\omega_{p \beta}^{2}}{\Omega_{*}^{2}} \frac{1}{n_{\beta 0}} J_{v 0}\left(f_{\beta 0}\right), \\
& \tilde{\mathrm{v}}_{c h}=\frac{a \Omega_{*}}{2 v_{*}} \sum_{\beta} \frac{\omega_{p \beta}^{2}}{\Omega_{*}^{2}} \frac{1}{n_{\beta 0}} J_{c h}\left(f_{\beta 0}\right) \\
& \tilde{\mathrm{v}}_{1}=-i \frac{a \Omega_{*}}{2 v_{*}} \sum_{\beta} \frac{\omega_{p \beta}^{2}}{\Omega_{*}^{2}} \frac{1}{n_{\beta 0}} \sum_{m=0}^{\infty} \sum_{n=-m}^{+m}\left(\frac{q_{\perp}}{r_{\beta}}\right)^{2 m} \\
& \times a(|n|, m-|n|) J_{U}\left(n, m, 0,1 ; f_{\beta 0}\right),
\end{aligned}
$$

where

$$
J_{U}\left(n, m, h, l ; f_{\beta 0}\right)=\int d^{3} u \frac{z\left(\tilde{\mathrm{v}}_{\beta d}^{0} / z\right)^{l} f_{\beta 0}}{z-n r_{\beta}-q_{\|} u_{\|}+i \tilde{\mathrm{v}}_{\beta d}^{0}}
$$

$$
\times \frac{u_{\|}^{h} u_{\perp}^{2 m}}{u} H\left(u^{2}+\frac{2 Z_{d 0} e q_{\beta}}{a m_{\beta} v_{*}^{2}}\right)
$$

$$
\begin{aligned}
& J_{v L}\left(n, m, h ; f_{\beta 0}\right)=\int d^{3} u \frac{\tilde{\mathrm{v}}_{\beta d}^{0} u_{\|}^{h} u_{\perp}^{2 m-1} L\left(f_{\beta 0}\right)}{z-n r_{\beta}-q_{\|} u_{\|}+i \tilde{\mathrm{v}}_{\beta d}^{0}}, \\
& J_{v v}\left(n, m ; f_{\beta 0}\right)=\int d^{3} u \frac{z\left(\tilde{\mathrm{v}}_{\beta d}^{0} / z\right)^{2} u_{\perp}^{2 m-1} \mathcal{L}\left(f_{\beta 0}\right)}{z-n r_{\beta}-q_{\|} u_{\|}+i \tilde{\mathrm{v}}_{\beta d}^{0}},
\end{aligned}
$$

$$
\begin{gathered}
J_{v 0}\left(f_{\beta 0}\right)=\int d^{3} u \frac{\tilde{\mathrm{v}}_{\beta d}^{0}}{z} \frac{\mathcal{L}\left(f_{\beta 0}\right)}{u_{\perp}}, \\
J_{c h}\left(f_{\beta 0}\right)=\int d^{3} u f_{\beta 0} \frac{1}{u} H\left(u^{2}+\frac{2 Z_{d 0} e q_{\beta}}{a m_{\beta} v_{*}^{2}}\right),
\end{gathered}
$$

with $\tilde{\mathrm{v}}_{1}=\mathrm{v}_{1} / \Omega_{*}$ and $\tilde{\mathrm{v}}_{c h}=\mathrm{v}_{c h} / \Omega_{*}$.

For the case of parallel propagation $\left(q_{\perp}=0\right)$, Eqs. (5), (8), and (9), lead to

$$
\begin{aligned}
e_{z z}= & -\frac{1}{z^{2}} \sum_{\beta} \frac{\omega_{p \beta}^{2}}{\Omega_{*}^{2}} \frac{1}{n_{\beta 0}} \int d^{3} u \frac{u_{\|}}{u_{\perp}} \mathcal{L}\left(f_{\beta 0}\right) \\
& +\frac{1}{z^{2}} \sum_{\beta} \frac{\omega_{p \beta}^{2}}{\Omega_{*}^{2}} \frac{1}{n_{\beta 0}}\left[J\left(0,0,2 ; f_{\beta 0}\right)+i J_{v}\left(0,0,1 ; f_{\beta 0}\right)\right], \\
\mathcal{U}_{z} & =\frac{1}{z} \frac{1}{z+i\left(\tilde{v}_{c h}+\tilde{v}_{1}\right)} \sum_{\beta} \frac{\omega_{p \beta}^{2}}{\Omega_{*}^{2}} \frac{1}{n_{\beta 0}} J_{U}\left(0,0,1,0 ; f_{\beta 0}\right), \\
S_{z}=- & \frac{a \Omega_{*}}{2 v_{*}} \frac{1}{z} \sum_{\beta} \frac{\omega_{p \beta}^{2}}{\Omega_{*}^{2}} \frac{1}{n_{\beta 0}}\left[J_{v L}\left(0,0,1 ; f_{\beta 0}\right)+i J_{v v}\left(0,0 ; f_{\beta 0}\right)\right] \\
+ & \frac{a \Omega_{*}}{2 v_{*}} \frac{1}{z} \sum_{\beta} \frac{\omega_{p \beta}^{2}}{\Omega_{*}^{2}} \frac{1}{n_{\beta 0}} J_{v 0}\left(f_{\beta 0}\right),
\end{aligned}
$$

where, from Eqs. (10) and (11),

$$
\begin{gathered}
\tilde{\mathrm{v}}_{c h}=\frac{a \Omega_{*}}{2 v_{*}} \sum_{\beta} \frac{\omega_{p \beta}^{2}}{\Omega_{*}^{2}} \frac{1}{n_{\beta 0}} J_{c h}\left(f_{\beta 0}\right), \\
\tilde{\mathrm{v}}_{1}=-i \frac{a \Omega_{*}}{2 v_{*}} \sum_{\beta} \frac{\omega_{p \beta}^{2}}{\Omega_{*}^{2}} \frac{1}{n_{\beta 0}} J_{U}\left(0,0,0,1 ; f_{\beta 0}\right) .
\end{gathered}
$$

Further development can be made in the particular case of bi-Maxwellian distributions for ions and electrons,

$$
f_{\beta 0}\left(u_{\|}, u_{\perp}\right)=\frac{n_{\beta 0}}{(2 \pi)^{3 / 2} u_{\beta \perp}^{2} u_{\beta \|}} e^{-u_{\|}^{2} /\left(2 u_{\beta \|}^{2}\right)} e^{-u_{\perp}^{2} /\left(2 u_{\beta \perp}^{2}\right)} .
$$

For these distributions,

$$
\mathcal{L}\left(f_{\beta 0}\right)=-\frac{u_{\|} u_{\perp}}{u_{\beta \perp}^{2}}\left(1-\Delta_{\beta}\right) f_{\beta 0},
$$


and

$$
L\left(f_{\beta 0}\right)=-\frac{u_{\perp}}{\gamma u_{\beta \perp}^{2}}\left[\gamma-\frac{q_{\|}}{z} u_{\|}\left(1-\Delta_{\beta}\right)\right] f_{\beta 0}
$$

where

$$
\Delta_{\beta}=\frac{u_{\beta \perp}^{2}}{u_{\beta \|}^{2}}=\frac{T_{\beta \perp}}{T_{\beta \|}} .
$$

where $u_{\beta \perp}=v_{\beta \perp} / v_{*}$ and $u_{\beta \|}=v_{\beta \|} / v_{*}$, with $v_{\beta \perp}=\sqrt{T_{\beta \perp} / m_{\beta}}$ and $v_{\beta \|}=\sqrt{T_{\beta \|} / m_{\beta}}$.

For the case of these distributions and using as an approximation the average value of the collision frequency instead of the actual momentum-dependent value, the integrals which are necessary for the components of the dielectric tensor can be evaluated, leading to the following expressions,

$$
\begin{gathered}
J\left(0,0,2 ; f_{\beta 0}\right)=(\sqrt{2})^{2} n_{\beta 0} \frac{u_{\beta \|}^{2}}{u_{\beta \perp}^{2}}\left\{\zeta_{\beta}^{0} \hat{\zeta}_{\beta}^{0}\left[1+\hat{\zeta}_{\beta}^{0} Z\left(\hat{\zeta}_{\beta}^{0}\right)\right]\right. \\
\left.-\left(1-\Delta_{\beta}\right)\left\{\frac{1}{2}+\left(\hat{\zeta}_{\beta}^{0}\right)^{2}\left[1+\hat{\zeta}_{\beta}^{0} Z\left(\hat{\zeta}_{\beta}^{0}\right)\right]\right\}\right\}, \\
J_{v}\left(0,0,1 ; f_{\beta 0}\right)=(\sqrt{2})^{2}\left(1-\Delta_{\beta}\right) n_{\beta 0} \\
\times \frac{u_{\beta \|}^{2}}{u_{\beta \perp}^{2}} \frac{\tilde{v}_{\beta}}{z} \zeta_{\beta}^{0} \hat{\zeta}_{\beta}^{0}\left[1+\hat{\zeta}_{\beta}^{0} Z\left(\hat{\zeta}_{\beta}^{0}\right)\right], \\
J_{U}\left(0,0,0,1 ; f_{\beta 0}\right) \simeq-\Gamma\left(\frac{1}{2}\right)(\sqrt{2})^{-1}\left(\frac{\tilde{v}_{\beta}}{z}\right)^{n_{\beta 0}} \\
\times\left(u_{\beta \perp}\right)^{-1}\left(u_{\beta \|}\right)^{0} \zeta_{\beta}^{0} Z\left(\hat{\zeta}_{\beta}^{0}\right), \\
J_{U}\left(0,0,1,0 ; f_{\beta 0}\right) \simeq-\Gamma\left(\frac{1}{2}\right)(\sqrt{2})^{0}\left(\frac{\tilde{v}_{\beta}}{z}\right)^{0} n_{\beta 0} \\
\times\left(u_{\beta \perp}\right)^{-1}\left(u_{\beta \|}\right)^{1} \zeta_{\beta}^{0}\left[1+\hat{\zeta}_{\beta}^{0} Z\left(\hat{\zeta}_{\beta}^{0}\right)\right] \\
J_{v v}\left(0,0 ; f_{\beta 0}\right)=\left(\frac{\tilde{v}_{\beta}}{z}\right)^{2}(\sqrt{2})^{1}\left(1-\Delta_{\beta}\right) n_{\beta 0}
\end{gathered}
$$

$$
\times\left(u_{\beta \perp}\right)^{-2}\left(u_{\beta \|}\right) \zeta_{\beta}^{0}\left[1+\hat{\zeta}_{\beta}^{0} Z\left(\hat{\zeta}_{\beta}^{0}\right)\right]
$$

$$
J_{v L}\left(0,0,1 ; f_{\beta 0}\right)=\frac{\tilde{v}_{\beta}}{z} J\left(0,0,1 ; f_{\beta 0}\right)=\frac{\tilde{v}_{\beta}}{z}(\sqrt{2})^{1} n_{\beta 0}
$$

$$
\times\left(u_{\beta \perp}\right)^{-2} u_{\beta \|}\left\{\zeta_{\beta}^{0}\left[1+\hat{\zeta}_{\beta}^{0} Z\left(\hat{\zeta}_{\beta}^{0}\right)\right]-\left(1-\Delta_{\beta}\right) \hat{\zeta}_{\beta}^{0}\left[1+\hat{\zeta}_{\beta}^{0} Z\left(\hat{\zeta}_{\beta}^{0}\right)\right]\right\}
$$

$$
J_{v 0}\left(f_{\beta 0}\right)=0
$$

where

$$
\begin{gathered}
\hat{\zeta}_{\beta}^{n}=\frac{z-n r_{\beta}+i \tilde{v}_{\beta}}{\sqrt{2} q_{\|} u_{\beta \|}}, \zeta_{\beta}^{0}=\frac{z}{\sqrt{2} q_{\|} u_{\beta \|}}, \\
\tilde{v}_{i}=\frac{v_{i}}{\Omega_{*}}=2(\sqrt{2 \pi})\left(\varepsilon n_{i 0}\right) \frac{c^{3}}{\Omega_{*}^{3}} \frac{a^{2} \Omega_{*}^{2}}{c^{2}} \frac{v_{*}}{c} \frac{u_{i \|}}{\Delta_{i}} \\
\times \int_{0}^{1} d \mu \frac{\Delta_{i}}{1+\mu^{2}\left(\Delta_{i}-1\right)}\left[\frac{\Delta_{i}}{1+\mu^{2}\left(\Delta_{i}-1\right)}+\chi_{\|}^{i}\right], \\
\tilde{v}_{e}=\frac{v_{e}}{\Omega_{*}}=2(\sqrt{2 \pi})\left(\varepsilon n_{i 0}\right) \frac{c^{3}}{\Omega_{*}^{3}} \frac{a^{2} \Omega_{*}^{2}}{c^{2}} \frac{v_{*}}{c} \frac{u_{e \|}}{\Delta_{e}} \\
\times \int_{0}^{1} d \mu\left[\frac{\Delta_{e}}{1+\mu^{2}\left(\Delta_{e}-1\right)}\right]^{2} e^{-\mid \chi_{\|}^{e}\left[11+\mu^{2}\left(\Delta_{e}-1\right)\right] / \Delta_{e}} .
\end{gathered}
$$

Moreover, the first integral which contributes to $e_{z z}$ becomes simply the following

$$
\int d^{3} u \frac{u_{\|}}{u_{\perp}} \mathcal{L}\left(f_{\beta 0}\right)=-\left(1-\Delta_{\beta}\right) n_{\beta 0} \frac{u_{\beta \|}^{2}}{u_{\beta \perp}^{2}} .
$$

Details of the evaluation can be found in appendix A. Using these results, and using also Eq. (A16) for the $J_{c h}$, the dispersion relation becomes

$$
\Lambda^{C}+\Lambda^{N}=0
$$

where

$$
\Lambda^{C}=1+\frac{2}{z^{2}} \sum_{\beta} \frac{\omega_{p \beta}^{2}}{\Omega_{*}^{2}} \zeta_{\beta}^{0} \hat{\zeta}_{\beta}^{0}\left[1+\hat{\zeta}_{\beta}^{0} Z\left(\hat{\zeta}_{\beta}^{0}\right)\right]
$$




$$
\begin{aligned}
\Lambda^{N} & =\frac{a \Omega_{*}}{2 v_{*}} \frac{\sqrt{2 \pi}}{z^{2}}\left[z+i \frac{a \Omega_{*}}{2 v_{*}} \sum_{\beta} \frac{\omega_{p \beta}^{2}}{\Omega_{*}^{2}}\left[\frac{1}{n_{\beta 0}} J_{c h}\left(f_{\beta 0}\right)+i \sqrt{\frac{\pi}{2}}\left(\frac{\tilde{v}_{\beta}}{z}\right) \frac{1}{u_{\beta \perp}} \zeta_{\beta}^{0} Z\left(\hat{\zeta}_{\beta}^{0}\right)\right]\right]^{-1} \\
& \times\left[\sum_{\beta} \frac{\omega_{p \beta}^{2}}{\Omega_{*}^{2}} \frac{u_{\beta} \|}{u_{\beta \perp}} \zeta_{\beta}^{0}\left[1+\hat{\zeta}_{\beta}^{0} Z\left(\hat{\zeta}_{\beta}^{0}\right)\right]\right]\left[\sum_{\beta} \frac{\omega_{p \beta}^{2}}{\Omega_{*}^{2}} \frac{u_{\beta \|}}{u_{\beta \perp}^{2}} \frac{\tilde{\mathrm{v}}_{\beta}}{z} \Delta_{\beta} \zeta_{\beta}^{0}\left[1+\hat{\zeta}_{\beta}^{0} Z\left(\hat{\zeta}_{\beta}^{0}\right)\right]\right] .
\end{aligned}
$$

\section{NUMERICAL ANALYSIS}

For the numerical analysis we consider parameters which are in the range of parameters of interest for stellar winds: ion temperature $T_{i}=1.0 \times 10^{4} \mathrm{~K}$, ion density $n_{i 0}=1.0 \times 10^{9}$ $\mathrm{cm}^{-3}$, ion charge number $Z_{i}=1.0$, and ion mass $m_{i}=m_{p}$, where $m_{p}$ is the proton mass, with $a=1.0 \times 10^{-4} \mathrm{~cm}$ as the radius of the dust particles. The ion density which has been assumed is rather high when compared, for instance, with the solar wind plasma, but is reported to occur in outbursts of carbonrich stars [17].

\subsection{Ion-acoustic waves, isotropic Maxwellian distributions}

Initially, we estimate the magnitude of the contribution of the 'new' terms to the dispersion relation of ES waves, and compare it with the 'conventional' contribution, for the case of isotropic Maxwellian distributions. In order to do that we assume the occurrence of weakly damped oscillations with frequency in the range of ion-acoustic waves, choosing the values $z=\left(1 \times 10^{-2},-2 \times 10^{-4}\right)$ for the numerical estimation. For this value of $z$ and for the parameters considered in the previous paragraph, and assuming $T_{e} / T_{i}=10.0$, we plot in Fig. 1 the quantities $\Lambda^{C}$ and $\Lambda^{N}$, namely the 'conventional' and the 'new' contributions to the ES dispersion relation, as defined in Eq. (20), versus normalized wave-number $q$ and normalized dust density $\varepsilon$. The upper panels of Fig. 1 show respectively, from left to right, the real and the imaginary parts of $\Lambda^{C}$, while the bottom panels show from left to right the real and the imaginary parts of $\Lambda^{N}$. It is seen that for most of the interval of $q$ and $\varepsilon$ depicted in the figure the real and imaginary contributions of $\Lambda^{N}$ are about four orders of magnitude smaller than the corresponding contributions of $\Lambda^{C}$. Similar figures and results can be obtained for different values of the ratio $T_{e} / T_{i}$, as in the cases of $T_{e} / T_{i}=1.0$ and $T_{e} / T_{i}=20.0$, which appeared in [9].

We further explore the role of the dust particles and of the 'new' contribution for the dispersion relation of ES waves in the case of isotropic Maxwellian distributions, by numerically solving the expanded form of Eq. (20) for the frequency range of ion-acoustic waves. Fig. 2 shows the value of $z_{r}$ and the corresponding values of the imaginary part $z_{i}$, as a function of $q$ and five values of $\varepsilon\left(0.0,2.5 \times 10^{-5}, 5.0 \times 10^{-5}, 7.5 \times 10^{-5}\right.$, and $1.0 \times 10^{-4}$ ), for three values of the temperature ratio $T_{e} / T_{i}$ $\left(1,10\right.$, and 20). Figure 2(a) shows that the quantity $z_{r}$ is relatively insensitive to the presence of the dust, for $T_{e}=T_{i}$. Figures 2(c) and 2(e) show that, for increasing values of the ratio $T_{e} / T_{i}$, the effect of the dust on the real part of the dispersion relation becomes more and more pronounced. Regarding the imaginary part, Figure 2(b) shows that Landau damping occurs for the whole range of $q$ values considered, namely the damping which occurs for absence of dust, $\varepsilon=0.0$, and that the presence of dust increases the damping for the whole range appearing in the figure, for $T_{e}=T_{i}$. On the other hand, Fig. 2(d) shows that for $T_{e} / T_{i}=10$ the increase of the dust population increases the damping in the region of very small $q$, and decreases the damping for sufficiently large $q(q \geq 0.008$, for the parameters utilized). There is an intermediate region for which the presence of the dust population initially contributes to decrease of damping, and then contributes to a renewed increase of damping, for sufficiently large $\varepsilon$. Similar features are seen more clearly with the increase of the ratio $T_{e} / T_{i}$. Figure 2(f) shows that for small $q$ the damping is appreciably increased with the increase of $\varepsilon$, while for $q \geq 0.015$ the damping is clearly decreased by the increase in the dust population.

The explanation for this behavior of the imaginary part $z_{i}$ is as follows. The basic feature to be considered is that the presence of dust lead to two competitive effects. One of the effects is the damping due to the dust charge variation, depending on the frequency of inelastic collisions on the denominator of the velocity integrals appearing in the dielectric tensor. Another effect is the reduction in the electron population, due to the capture of electrons by the dust particles, which contribute to reduction of electron Landau damping. For $T_{e} / T_{i}=1.0$ the electron Landau damping is meaningful for large $q$, and decreases for small $q$, because the resonant velocity becomes much larger than the electron thermal velocity. The dust charge variation constitutes an additional damping mechanism. This effect dominates over the decrease of Landau damping because the capture of electrons is not very significant for $T_{e} / T_{i}=1.0$. Figure 3 shows that for $T_{e} / T_{i}=1.0$ and $\varepsilon=1.0 \times 10^{-4}$ the electron density is still more than $80 \%$ of the population in a dustless plasma. The consequence is that the damping is increased by the process of dust charge variation, for the whole range of $q$, with the increase of the dust density. For $T_{e} / T_{i}=10.0$, on the other hand, electron Landau damping is significant for the higher end of the $q$ range considered, but less important for small $q$, if compared with the case of $T_{e} / T_{i}=1.0$. With the increase in the dust population, the damping is enhanced for small $q$, since in the region of small Landau damping the damping due to the dust charge variation is dominant. This possibility of damping due to collisional charging has already been noticed by other authors $[3,18]$. In the higher end of the $q$ region, however, Landau damping is sufficiently high to become dominant. Although the presence of dust introduces damping due to the dust charge variation, the dominant effect is the reduction of Landau damping due to the reduction of the electron population. Figure 3 shows that for $T_{e} / T_{i}=10.0$ the electron density is reduced to nearly $30 \%$ of the original density, for the largest value $\varepsilon$ considered in the calculation. These 
features are even more evident for $T_{e} / T_{i}=20.0$. For small $q$ the Landau damping is negligible in this case. With the increase of the dust population, there is enhancement of damping for small $q$, due to the mechanism of dust charge variation. For the higher end of the $q$ region, however, the damping due to the dust charge variation is overcome by Landau damping. With the increase of the dust population $\varepsilon$, the electron population is severely reduced, as shown by Fig. 3, and the overall effect is the reduction of the wave damping shown by Fig. 2(f).

We point out that in Fig. 2 we have plotted the results obtained with the dispersion relation given by Eq. (20). We have also plotted in the same figure the results obtained from a dispersion relation given by $\Lambda^{C}=0$, obtained by neglecting the 'new' contribution to the dielectric tensor. The results hardly can be distinguished in the scale of the figure, reflecting the fact that for the range of frequency and for the parameters utilized the effect of the 'new' contribution is negligible in the dispersion relation of ES waves. In a color version of Fig. 2, using blue color for the results obtained with the full dispersion relation and red color for the results obtained considering only the "conventional" contribution to the dispersion relation, the two different results appear so close that the curves feature a light purple color, result of the superposition of the results featured with blue color and the results featured with red color. In a monochromatic version of Fig. 2, using two different line styles, the two different results can hardly be distinguished.

\subsection{Ion-acoustic waves, bi-Maxwellian distributions}

At this point we proceed to the estimation of the magnitude of the contribution of the 'new' terms to the dispersion relation of ES waves in anisotropic plasmas, and compare it with the 'conventional' contribution. In order to do that we again assume the occurrence of weakly damped oscillations with frequency in the range of the ion-acoustic waves, assuming a typical normalized frequency $z=\left(1.0 \times 10^{-2},-2 \times 10^{-4}\right)$. For this value of $z$ and for the parameters considered in the previous paragraph, and assuming $T_{e} / T_{i}=1.0$, and considering the case of $T_{e \perp} / T_{e \|}=0.1$ and $T_{i \perp} / T_{i \|}=0.1$, we plot in Fig. 4 the quantities $\Lambda^{C}$ and $\Lambda^{N}$ versus normalized wave-number $q$ and normalized dust density $\varepsilon$. The upper panels of Fig. 4 show respectively, from left to right, the real and the imaginary parts of $\Lambda^{C}$, while the bottom panels show from left to right the real and the imaginary parts of $\Lambda^{N}$. It is seen that for most of the interval of $q$ and $\varepsilon$ depicted in the figure the real and imaginary contributions of $\Lambda^{N}$ are much smaller than the corresponding contributions of $\Lambda^{C}$, similarly to what occurs in the case of isotropic Maxwellian distributions.

In Fig. 5 we show the same quantities depicted in Fig. 4, for the case of $T_{e \perp} / T_{e \|}=10.0$ and $T_{i \perp} / T_{i \|}=10.0$, with the other parameters all equal to those used for Fig. 1. The comments which can be made about Fig. 5 are similar to those made about Fig. 4, and also similar to those made about Fig. 1, which was obtained for the case of isotropy of temperatures.

Figures 4 and 5 have been obtained assuming equal ion and electron temperatures. In the case of electron temperature larger than ion temperature, similar results can be obtained. The only point to be observed is that, for increasing ratio of perpendicular and parallel temperatures, the magnitudes of $\Lambda^{C}$ and $\Lambda^{N}$ at small $q$, which are seen to grow with $\varepsilon$ in the range depicted in Figs. 4 and 5 , are seen to decrease again at sufficiently large $\varepsilon$. This feature is illustrated in Figs. 6 and 7, which show the same quantities appearing in Figs. 4 and 5 , for $T_{e} / T_{i}=4.0$ and $T_{e} / T_{i}=10.0$, respectively, with $T_{e \perp} / T_{e \|}=10.0$ and $T_{i \perp} / T_{i \|}=10.0$, and the other parameters all equal to those used for Fig. 1. The opposite side of the anisotropy range is illustrated by Fig. 8, which shows the case of $T_{e} / T_{i}=10.0$, with $T_{e \perp} / T_{e \|}=0.1$ and $T_{i \perp} / T_{i \|}=0.1$, and the other parameters all equal to those used for Fig. 1.

Both in the case of $\Delta_{\beta}=0.1$, shown in Fig. 4 , in the case of $\Delta_{\beta}=10.0$, shown in Figs. 5 and 6 , and in the case of $\Delta_{\beta}=1.0$, illustrated in Fig. 1, the comparison between the magnitude of the 'conventional' and 'new' contribution becomes more difficult in the region of the graphics where these contributions both approach zero. In order to improve the accuracy of observation, we show in Fig. 9 the ratio between the 'new' and the 'conventional' contributions, for one of the cases discussed. In the upper line of Fig. 9 we show at the left-hand side the ratio between the real parts of the contributions, and in the righthand side the ratio between the imaginary parts, for the case $T_{e \perp} / T_{e \|}=0.1$ and $T_{i \perp} / T_{i \|}=0.1$, with $T_{e} / T_{i}=1.0$, and other parameters as in Fig. 1. At the bottom line, we show the corresponding figures for the case of perpendicular temperatures much above parallel temperatures, with $T_{e \perp} / T_{e \|}=10.0$ and $T_{i \perp} / T_{i \|}=10.0$, with $T_{e} / T_{i}=1.0$, and other parameters as in Fig. 1.

The conclusion to be drawn from Fig. 1, for the case of plasmas with isotropy of temperature, and from Figs. 4, 5, 6, and 9 is that, although the dust population may introduce significant contribution to the dispersion relation of electrostatic waves in the range of frequencies characteristics of ion-acoustic waves, this contribution is mostly due to the 'conventional' part of the dielectric tensor. At least for the parameter regime which has been investigated, the 'new' contribution is shown to give only a negligible contribution to the dispersion relation.

We continue with the investigation of the role played by the dust particles and by the 'new' contribution for the dispersion relation of ES waves in anisotropic plasmas, by discussing the numerical solution of the dispersion relation. In Fig. 10 we consider the solution corresponding to ion-acoustic waves, also for three situations of temperature anisotropy. Fig. 10(a) shows the value of $z_{r}$ for ion-acoustic waves, as a function of $q_{z}$ and five values of $\varepsilon\left(0.0,2.5 \times 10^{-5}, 5.0 \times 10^{-5}, 7.5 \times 10^{-5}\right.$, and $\left.1.0 \times 10^{-4}\right)$, for $T_{e}=T_{i}$ and perpendicular temperature much smaller than parallel temperature $\left(T_{e \perp} / T_{e \|}=T_{i \perp} / T_{i \|}=\right.$ 0.1 ), with other parameters as in Fig. 1. Figure 10(b) shows the corresponding values of the imaginary part of the normalized frequency, $z_{i}$. It is is seen that the presence of the dust population modifies very significantly the imaginary part $z_{i}$. The damping, measured by the absolute value of $z_{i}$, is enhanced for small $q_{z}$, due to the presence of the dust, but can be appreciably reduced for larger $q_{z}$, also due to the presence of the dust. For the real part $z_{r}$, Fig. 10(c) shows that the effect of the dust is not very significant, although not negligible.

The case of perpendicular temperature larger than the parallel temperature is seen in Figs. 10(e) and 10(f), at the bottom line of Fig. 10. Figure 10(e) shows the value of $z_{r}$ for ionacoustic waves, as a function of $q_{z}$ and five values of $\varepsilon(0.0$, $2.5 \times 10^{-5}, 5.0 \times 10^{-5}, 7.5 \times 10^{-5}$, and $\left.1.0 \times 10^{-4}\right)$, for $T_{e}=T_{i}$ and $T_{e \perp} / T_{e \|}=T_{i \perp} / T_{i \|}=10.0$, with other parameters as in Fig. 1. Figure 10(e) shows that in this case of larger perpendicular 

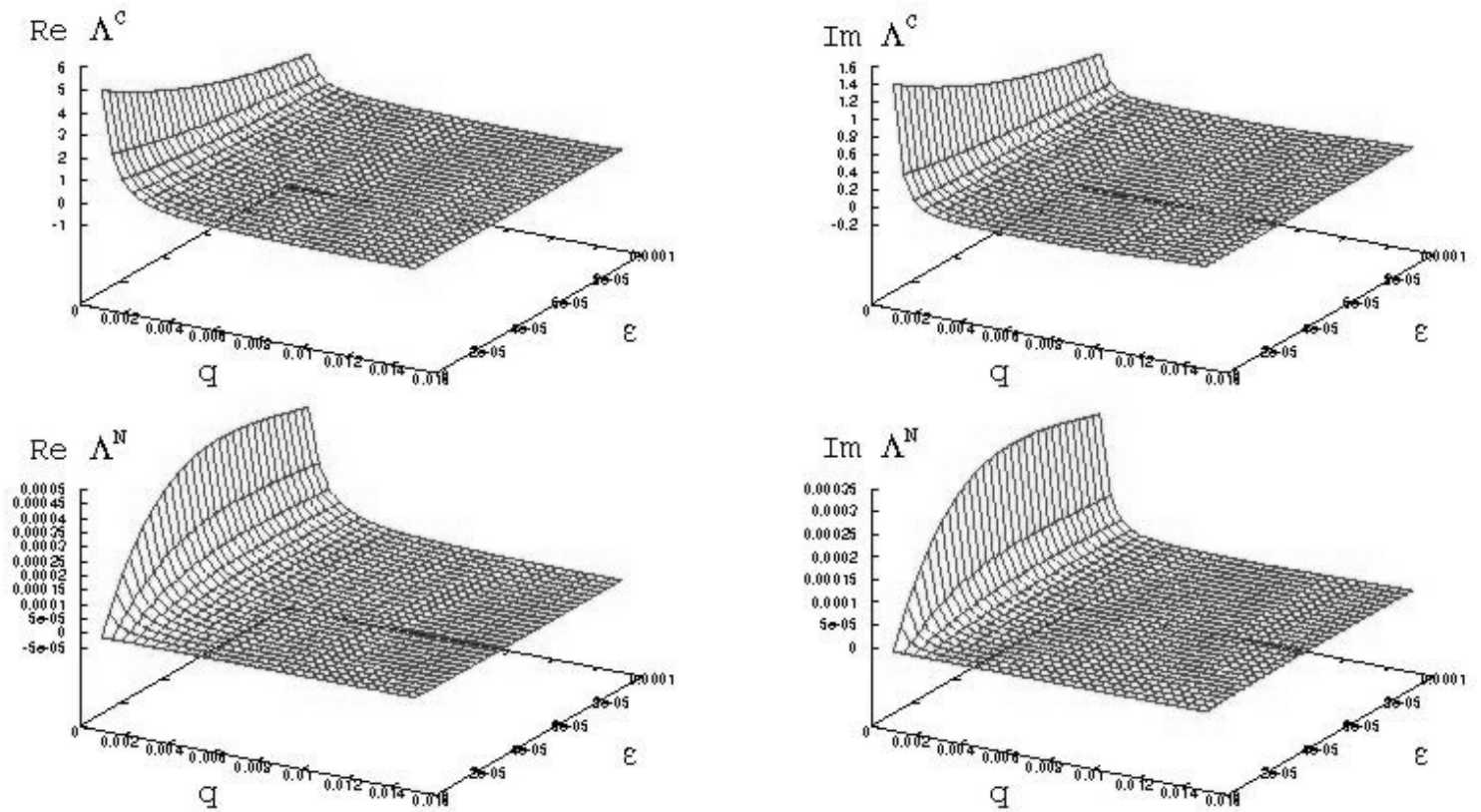

FIG. 1: (upper left) Real part of the "conventional" contribution to the dispersion relation, vs. $q$ and $\varepsilon=n_{d} / n_{i 0}$; (upper right) imaginary part of the "conventional" contribution; (bottom left) Real part of the "new" contribution; (bottom right) imaginary part of the "new" contribution; $z=\left(1.0 \times 10^{-2},-2.0 \times 10^{-4}\right)$, in the range of ion-acoustic waves. Isotropic Maxwellian distributions for ions and electrons, with $T_{i}=1.0 \times 10^{4}$ $\mathrm{K}$ and $T_{e} / T_{i}=10$. Other parameters: $n_{i 0}=1.0 \times 10^{9} \mathrm{~cm}^{-3}, Z_{i}=1.0, m_{i}=m_{p}$, where $m_{p}$ is the proton mass, and $a=1.0 \times 10^{-4} \mathrm{~cm}$.

temperature the real part of the normalized frequency is much more affected by the presence of the dust than in the case of smaller perpendicular temperature. The imaginary part $z_{i}$ is also affected significantly by the presence of the dust population, as shown by Fig. 10(f). Qualitatively, it is seen that the effect is similar to that occurring in the case of perpendicular temperature smaller than parallel shown in Fig. 10(b), in the sense that the damping, measured by the absolute value of $z_{i}$, is enhanced for small $q_{z}$, due to the presence of the dust, but can be appreciably reduced for larger $q_{z}$, also due to the presence of the dust.

The middle line of Fig. 10 shows the case of isotropy of temperatures, in between the cases depicted at the top line and at the bottom line. Figures 10(c) and 10(d) show the values of $z_{r}$ and $z_{i}$, respectively, for ion-acoustic waves, as a function of $q_{z}$ and five values of $\varepsilon\left(0.0,2.5 \times 10^{-5}, 5.0 \times 10^{-5}, 7.5 \times 10^{-5}\right.$, and $\left.1.0 \times 10^{-4}\right)$, for $T_{e}=T_{i}$ and $T_{e \perp} / T_{e \|}=T_{i \perp} / T_{i \|}=1.0$, with other parameters as in Fig. 1 .

Additional information may be obtained by considering the dependence of the electron density and of the equilibrium value of the charge of the dust particles, as a function of the dust density. In Fig. 11 we show the values of the dust charge $Z_{d 0}$ and of the electron density $n_{e 0}$ as a function of $\varepsilon$, for five values of the ratio $T_{\perp} / T_{\|}$, considering $T_{e \|}=20 T_{i \|}$, for fixed ion density, for the case of ion-sound waves. It is seen that for $T_{\perp}=0.2 T_{\|}$the value of $Z_{d 0}$ reduced by nearly $30 \%$ when $\varepsilon$ is changed between 0.0 and $1.0 \times 10^{-4}$, while it is reduced in the same range to nearly $15 \%$ of the original value in the case of $T_{\perp}=5.0 T_{\|}$. It is also seen that the value of $n_{e 0}$ changes by approximately $60 \%$ when $\varepsilon$ is changed between 0.0 and $1.0 \times 10^{-4}$, in the case of $T_{\perp} / T_{\|}=0.2$, and is reduced to almost $5 \%$ of the original value in the case $T_{\perp} / T_{\|}=5.0$.

The dependence of $Z_{d 0}$ and $n_{e 0}$ on the ratio of electron and ion temperatures, for anisotropic situations, is illustrated in Fig. 12. Figure 12(a) shows the values of $Z_{d 0}$ as a function of $\varepsilon$, for four values of the ratio $T_{e \|} / T_{i \|}$, for a case with anisotropy of temperatures, with $T_{\perp} / T_{\|}=5.0$. Figure 12(b) shows the values of the ratio $n_{e 0}(\varepsilon) / n_{e 0}(0)$, as a function of $\varepsilon$, for four values of the ratio $T_{e \|} / T_{i \|}$, for $T_{\perp} / T_{\|}=5.0$. It is seen that the density of electrons decrease with the increase of $\varepsilon$, substantially faster for the case of high values of the ratio $T_{e \|} / T_{i \|}$ than for the case in which this ratio is equal to the unity.

\subsection{Langmuir waves, bi-Maxwellian distributions}

We proceed by estimating the magnitude of the contribution of the 'new' terms to the dispersion relation of ES waves in anisotropic plasmas, now considering the case of waves with frequency in the range of the Langmuir waves, assuming a typical normalized frequency $z=\left(1.1 \times 10^{0},-1 \times 10^{-3}\right)$. For this value of $z$ and for the parameters considered in the previous paragraph, and assuming $T_{e} / T_{i}=1.0$, and considering the case of $T_{e \perp} / T_{e \|}=0.1$ and $T_{i \perp} / T_{i \|}=0.1$, we plot in Fig. 13 the quantities $\Lambda^{C}$ and $\Lambda^{N}$, namely the 'conventional' and the 'new' contributions to the ES dispersion relation, as defined in Eq. (20), versus normalized wave-number $q$ and normalized dust density $\varepsilon$. The upper panels of Fig. 13 show respectively, 

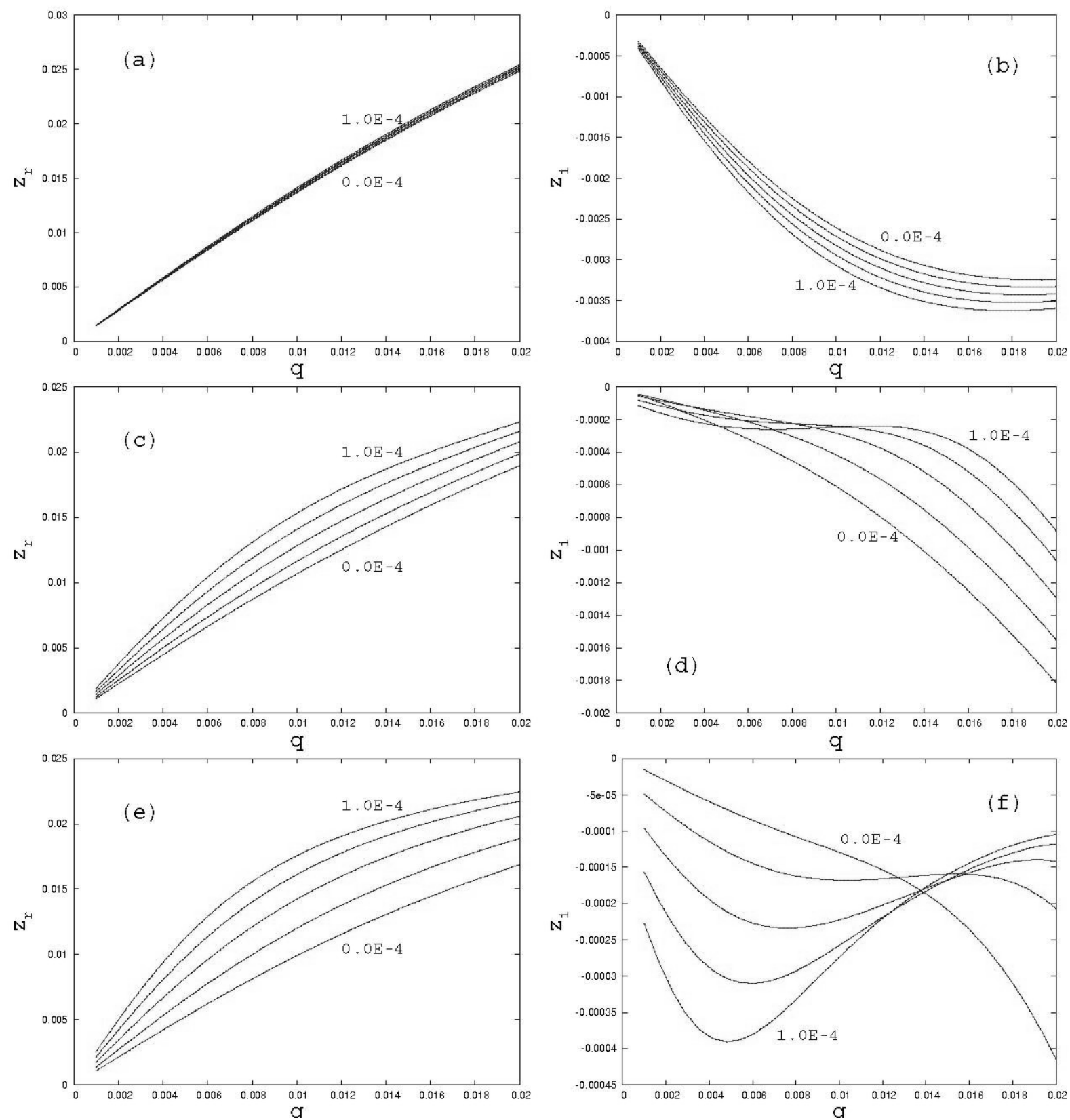

FIG. 2: Real and imaginary parts of the normalized frequency $\left(z_{r}\right.$ and $\left.z_{i}\right)$ obtained from the dispersion relation for ion-acoustic waves in the case of isotropic Maxwellian distributions for ions and electrons, vs. $q$, for several values of $\varepsilon=n_{d} / n_{i 0}\left(0.0,2.5 \times 10^{-5}, 5.0 \times 10^{-5}, 7.5 \times 10^{-5}\right.$, and $1.0 \times 10^{-4}$ ); (a) $z_{r}$, with $T_{e} / T_{i}=1.0$; (b) $z_{i}$, with $T_{e} / T_{i}=1.0$; (c) $z_{r}$, with $T_{e} / T_{i}=10.0$; (d) $z_{i}$, with $T_{e} / T_{i}=10.0$; (e) $z_{r}$, with $T_{e} / T_{i}=20.0$; (f) $z_{i}$, with $T_{e} / T_{i}=20.0$; other parameters the same as used to obtain Fig. 1.

from left to right, the real and the imaginary parts of $\Lambda^{C}$, while the bottom panels show from left to right the real and the imaginary parts of $\Lambda^{N}$. It is seen that for most of the interval of $q$ and $\varepsilon$ depicted in the figure the real and imaginary contributions of $\Lambda^{N}$ are several orders of magnitude smaller than the corresponding contributions of $\Lambda^{C}$.

In Fig. 14 we show the same quantities depicted in Fig. 13, for the case of $T_{e \perp} / T_{e \|}=10.0$ and $T_{i \perp} / T_{i \|}=10.0$, with the other parameters all equal to those used for Fig. 13. The comments which can be made about Fig. 14 are similar to those made about Fig. 13, and also similar to those made about Fig. 1 of Ref. [9], where the case of isotropic temperatures has been discussed. The conclusion is that the dust population may introduce significant contribution to the dispersion relation of electrostatic waves in the range of frequencies characteristics of Langmuir waves, but this contribution is mostly due to the 'conventional' part of the dielectric tensor. At least for the parameter regime which has been investigated, the 'new' contribution is shown to give only a negligible contribution to the dispersion relation.

In Fig. 15 we consider the solution corresponding to Langmuir waves, for three situations of temperature anisotropy. Fig. 15(a) shows the value of $z_{r}$ as a function of $q_{z}$ and five values of $\varepsilon\left(0.0,2.5 \times 10^{-5}, 5.0 \times 10^{-5}, 7.5 \times 10^{-5}\right.$, and $\left.1.0 \times 10^{-4}\right)$, 


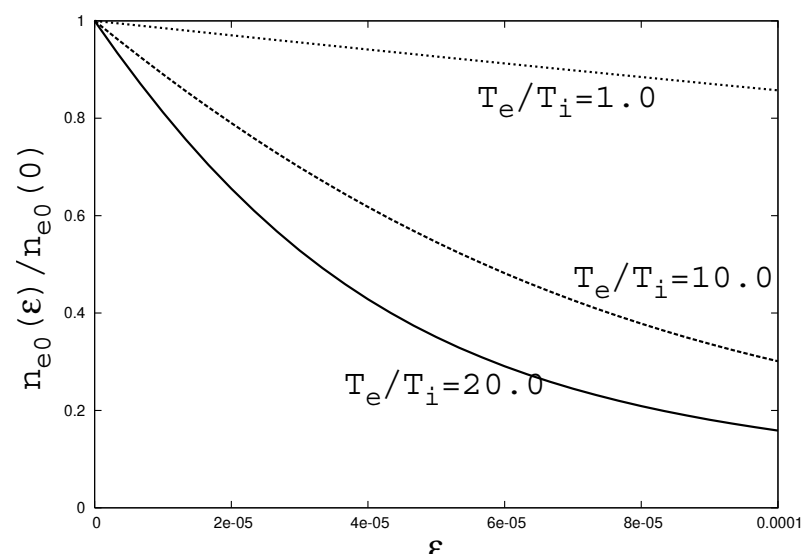

FIG. 3: Ratio between the electron density in a dusty plasma and the equilibrium electron density in the absence of dust, vs. $\varepsilon=n_{d} / n_{i 0}$, for three values of the ratio $T_{e} / T_{i}$, in the case of isotropic Maxwellian distributions for ions and electrons, Other parameters the same as used to obtain Fig. 1.
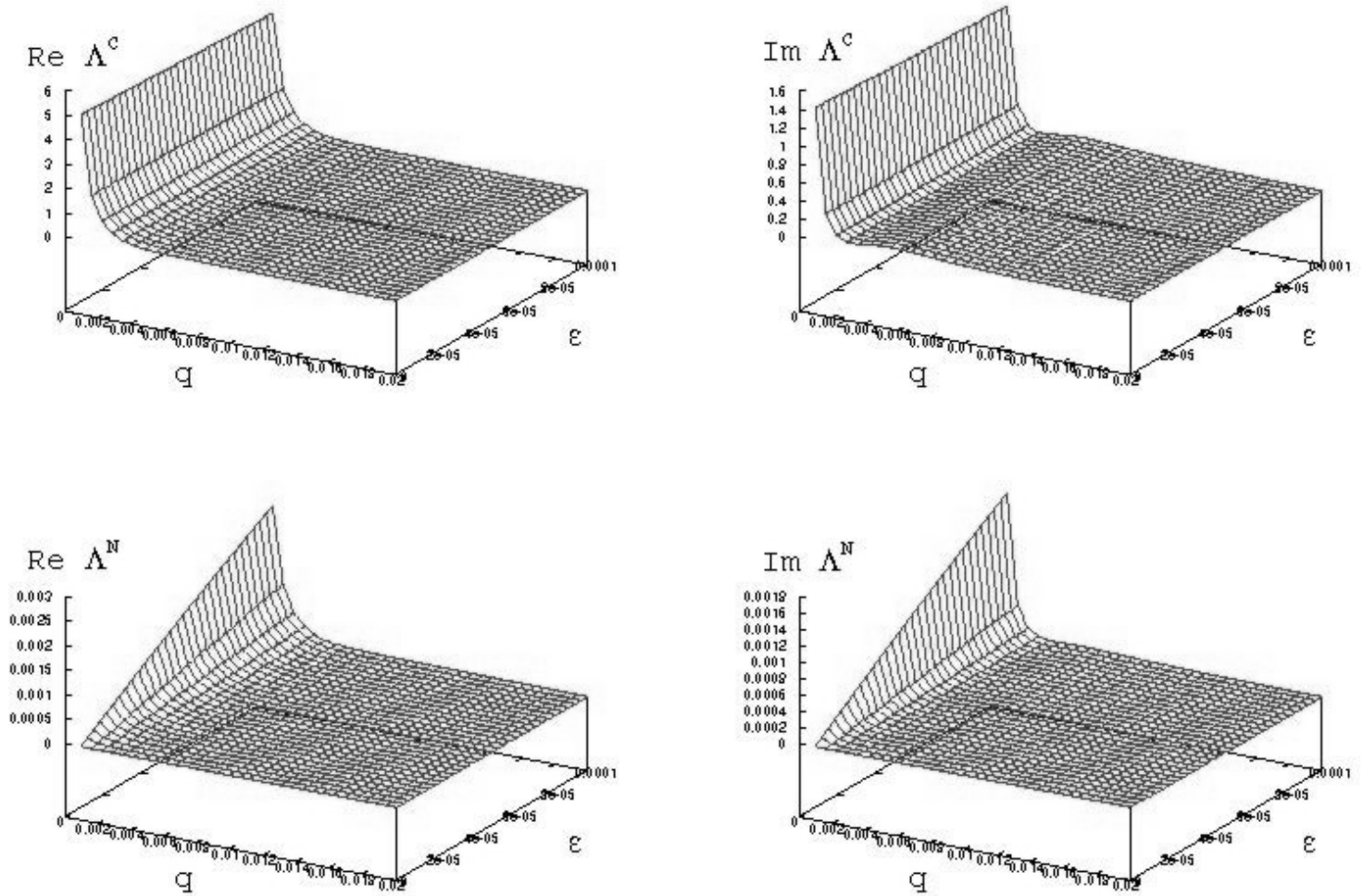

FIG. 4: (upper left) Real part of the 'conventional' contribution to the dispersion relation, vs. $q$ and $\varepsilon=n_{d} / n_{i 0}$; (upper right) imaginary part of the 'conventional' contribution; (bottom left) Real part of the 'new' contribution; (bottom right) imaginary part of the 'new' contribution; $T_{e}=T_{i}, \Delta_{\beta}=T_{\beta \perp} / T_{\beta \|}=0.1$, for $\beta=i, e$, and $z=\left(1.0 \times 10^{-2},-2.0 \times 10^{-4}\right)$, in the range of ion-acoustic waves. Other parameters the same as used to obtain Fig. 1 .

for $T_{e}=T_{i}$ and perpendicular temperature much smaller than parallel temperature $\left(T_{e \perp} / T_{e \|}=T_{i \perp} / T_{i \|}=0.1\right)$, with other parameters as in Fig. 13. Figure 15(b) shows the corresponding values of the imaginary part of the normalized frequency, $z_{i}$. It is is seen that the presence of the dust population do not modifies appreciably the root of the dispersion relation, either the real or the imaginary part.

Figure 15(e) shows the value of $z_{r}$ for Langmuir waves, as a function of $q_{z}$ and five values of $\varepsilon\left(0.0,2.5 \times 10^{-5}\right.$, $5.0 \times 10^{-5}, 7.5 \times 10^{-5}$, and $\left.1.0 \times 10^{-4}\right)$, for $T_{e}=T_{i}$ and perpendicular temperature much larger than parallel temperature $\left(T_{e \perp} / T_{e \|}=T_{i \perp} / T_{i \|}=10.0\right)$, with other parameters as in Fig. 

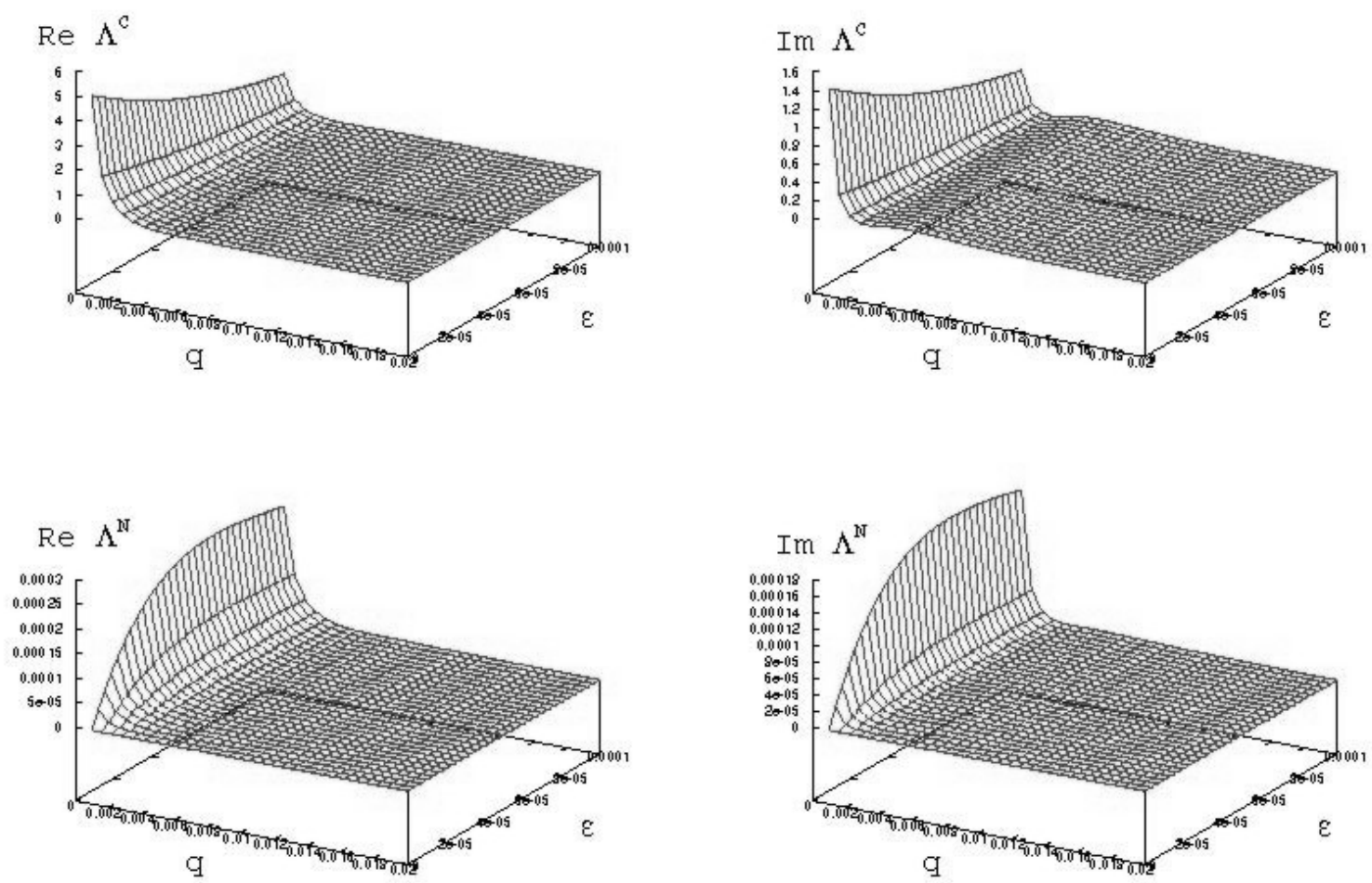

FIG. 5: (upper left) Real part of the 'conventional' contribution to the dispersion relation, vs. $q$ and $\varepsilon=n_{d} / n_{i 0}$; (upper right) imaginary part of the 'conventional' contribution; (bottom left) Real part of the 'new' contribution; (bottom right) imaginary part of the 'new' contribution; $T_{e}=T_{i}, \Delta_{\beta}=T_{\beta \perp} / T_{\beta \|}=10.0$, for $\beta=i, e$, and $z=\left(1.0 \times 10^{-2},-2.0 \times 10^{-4}\right)$, in the range of ion-acoustic waves. Other parameters the same as used to obtain Fig. 1 .

13. Figure 15(e) shows that in this case of larger perpendicular temperature the real part of the normalized frequency is much more affected by the presence of the dust than in the case of smaller perpendicular temperature. Particularly in the region of small wave-number ( $\operatorname{cmall} q_{z}$ ), the value of $z_{r}$ can be reduced by a factor which goes up to almost $50 \%$, for dust population rising between the case $\varepsilon=0.0$ and the case $\varepsilon=1.0 \times 10^{-4}$. Figure 15 (f) shows the corresponding values of the imaginary part of the normalized frequency, $z_{i}$. Contrarily to what happens in the case in which the perpendicular temperature is smaller than the parallel temperature, shown in Fig. 15(b), Fig. 15(f) shows that in the case of larger perpendicular temperature the value of the imaginary part $z_{i}$ can be very appreciably affected by the presence of the dust population. For a given value of the normalized wave number $q_{z}$, the magnitude of the imaginary part of $z_{i}$ is significantly increased by the presence of the dust.

In between the cases shown at the top line and at the bottom line of Fig. 15, we consider the case of equal parallel and perpendicular temperatures. Figure 15(c) shows the value of $z_{r}$ for Langmuir waves, as a function of $q_{z}$ and five values of $\varepsilon$ $\left(0.0,2.5 \times 10^{-5}, 5.0 \times 10^{-5}, 7.5 \times 10^{-5}\right.$, and $\left.1.0 \times 10^{-4}\right)$, for $T_{e}=T_{i}$ and perpendicular temperature equal to parallel temperature $\left(T_{e \perp} / T_{e \|}=T_{i \perp} / T_{i \|}=1.0\right)$, with other parameters as in Fig. 13. Figure 15(d) shows the corresponding values of the imaginary part of the normalized frequency, $z_{i}$.
Let us now consider the dependence of the electron density and of the equilibrium value of the charge of the dust particles, as a function of the dust density. In Fig. 16 we show the values of the dust charge $Z_{d 0}$ and of the electron density $n_{e 0}$ as a function of $\varepsilon$, for five values of the ratio $T_{\perp} / T_{\|}$, considering $T_{e \|}=T_{i \|}$, for fixed ion density. The case of $T_{e \|}=T_{i \|}$ is the case relevant for Langmuir waves. It is seen that the value of $Z_{d 0}$ is basically independent of $\varepsilon$ in the range considered, for $T_{\perp}<T_{\|}$, changing by less than $5 \%$ when $\varepsilon$ is changed between 0.0 and $1.0 \times 10^{-4}$, in the case $T_{\perp}=T_{\|}$. For larger values of this ratio the value of $Z_{d 0}$ becomes more dependent on $\varepsilon$, although even for $T_{\perp} / T_{\|}=5.0$ the change obtained is only of order of $20 \%$, in the range considered. It is also seen that the value of $n_{e 0}$ changes by approximately $8 \%$ when $\varepsilon$ is changed between 0.0 and $1.0 \times 10^{-4}$, in the case of $T_{\perp} / T_{\|}=0.2$, and is reduced to almost $50 \%$ of the original value in the case $T_{\perp} / T_{\|}=5.0$. These results show that the electron density and the dust charge are more and more sensitive to the dust density for increasing values of the ratio $T_{\perp} / T_{\|}$, for fixed ion density.

\section{CONCLUSIONS}

In the present paper we have examined the influence of the dust charge variation on the dispersion relation of electrostatic waves, considering the case of waves propagating in the direc- 

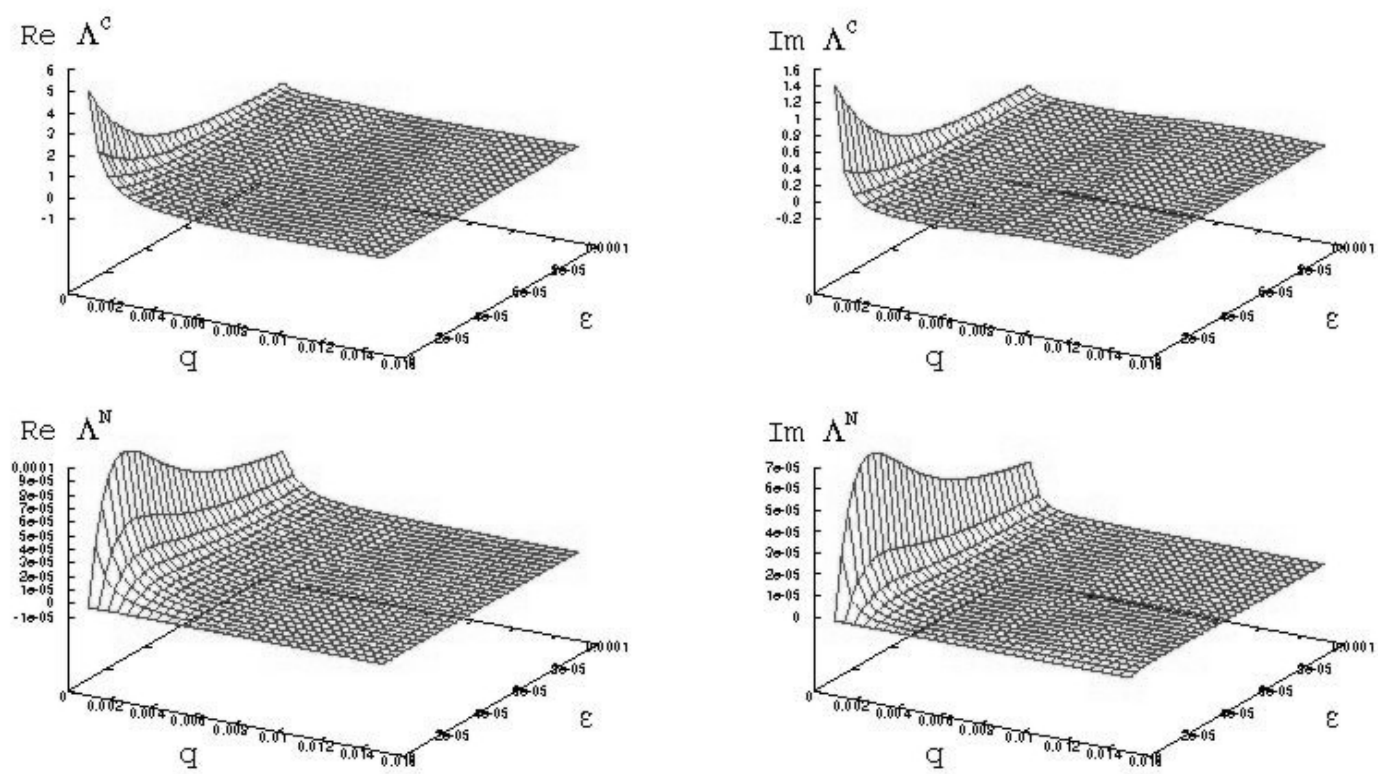

FIG. 6: (upper left) Real part of the 'conventional' contribution to the dispersion relation, vs. $q$ and $\varepsilon=n_{d} / n_{i 0}$; (upper right) imaginary part of the 'conventional' contribution; (bottom left) Real part of the 'new' contribution; (bottom right) imaginary part of the 'new' contribution; $T_{e} / T_{i}=4.0, \Delta_{\beta}=T_{\beta \perp} / T_{\beta \|}=10.0$, for $\beta=i, e$, and $z=\left(1.0 \times 10^{-2},-2.0 \times 10^{-4}\right)$, in the range of ion-acoustic waves. Other parameters the same as used to obtain Fig. 1.
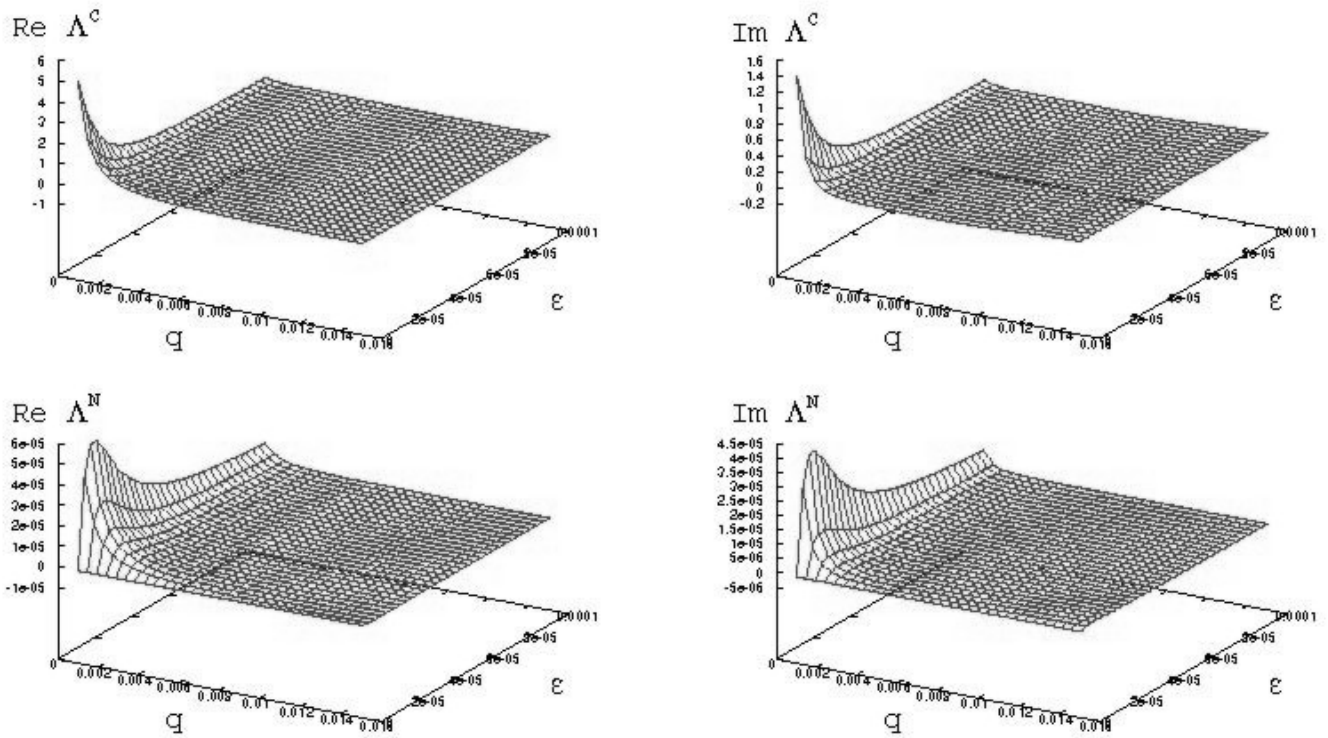

FIG. 7: (upper left) Real part of the 'conventional' contribution to the dispersion relation, vs. $q$ and $\varepsilon=n_{d} / n_{i 0}$; (upper right) imaginary part of the 'conventional' contribution; (bottom left) Real part of the 'new' contribution; (bottom right) imaginary part of the 'new' contribution; $T_{e} / T_{i}=10.0, \Delta_{\beta}=T_{\beta \perp} / T_{\beta \|}=10.0$, for $\beta=i, e$, and $z=\left(1.0 \times 10^{-2},-2.0 \times 10^{-4}\right)$, in the range of ion-acoustic waves. Other parameters the same as used to obtain Fig. 1.

tion of the ambient magnetic field. The most important motivation was the investigation of the effect of some contributions to the dielectric tensor which usually appear in theoretical analysis, but are commonly neglected in numerical analysis.

For the dielectric tensor, we have used a kinetic formulation which takes into account the incorporation of electrons and ions to the dust particles due to inelastic collisions, shaped in a form in which the components of the dielectric tensor are written in terms of double summations, related to harmonic and Larmor radius contributions. The general expressions utilized depend on a small number of integrals which depend on the distribution function. 

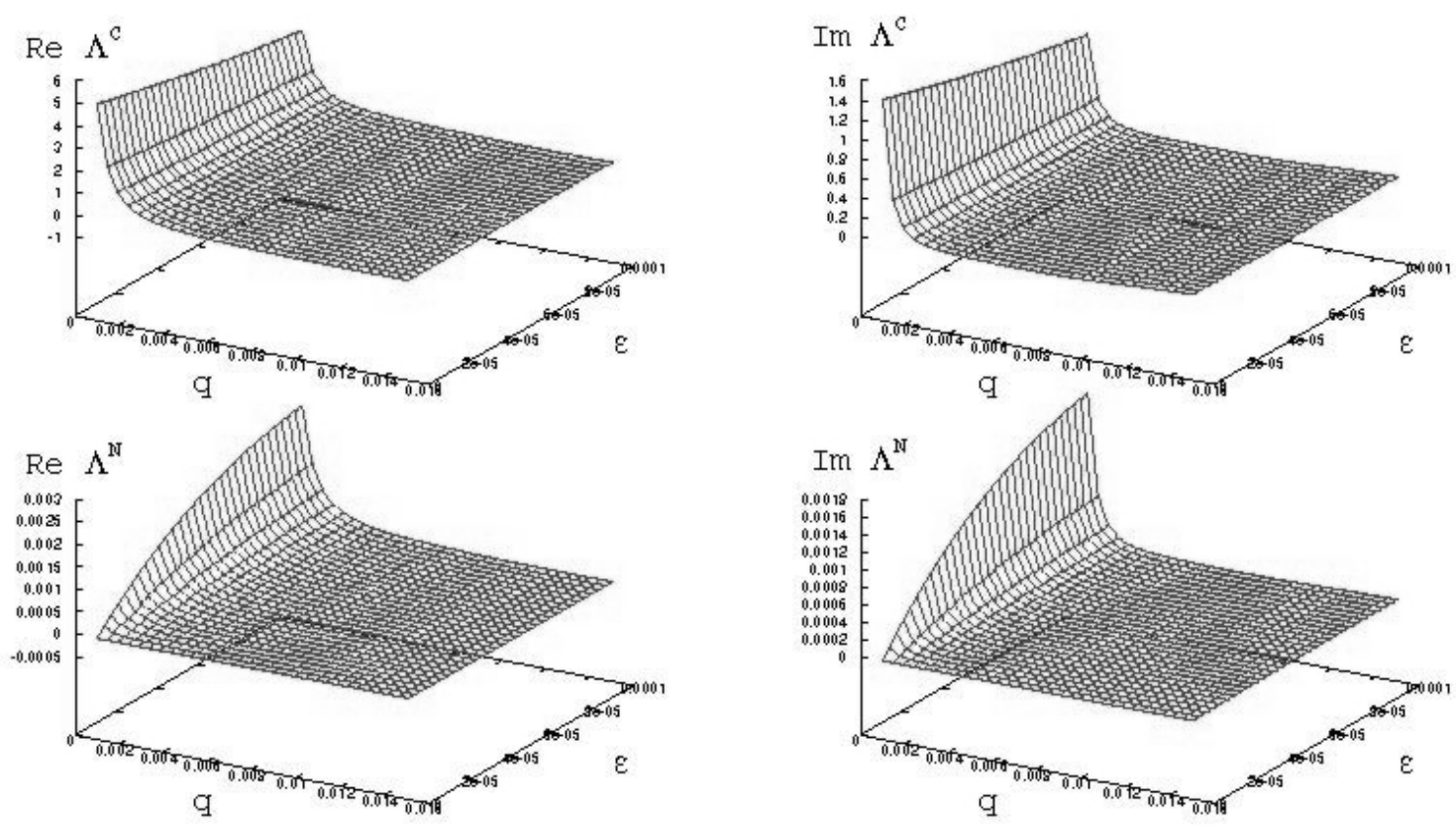

FIG. 8: (upper left) Real part of the 'conventional' contribution to the dispersion relation, vs. $q$ and $\varepsilon=n_{d} / n_{i 0}$; (upper right) imaginary part of the 'conventional' contribution; (bottom left) Real part of the 'new' contribution; (bottom right) imaginary part of the 'new' contribution; $T_{e} / T_{i}=10.0, \Delta_{\beta}=T_{\beta \perp} / T_{\beta \|}=0.1$, for $\beta=i, e$, and $z=\left(1.0 \times 10^{-2},-2.0 \times 10^{-4}\right)$, in the range of ion-acoustic waves. Other parameters the same as used to obtain Fig. 1 .

One interesting point is that the dielectric tensor can be divided into two parts. One of these parts is denominated 'conventional', and is formally similar to the dielectric tensor of dustless plasmas, modified by the presence of a collision frequency related to the inelastic collision between dust particles and plasma particles. The other part owes its existence to the occurrence of the dust charge variation, and is denominated as the 'new' contribution.

We have considered the case of anisotropic Maxwellian distributions for ions and electrons, and introduced an approximation which uses the average value of the inelastic collision frequencies of electrons and ions with the dust particles, instead of the actual momentum dependent expressions. This approximation was adopted in order to arrive at a relatively simple estimate of the effect of the dust charge variation, effect which is frequently neglected in analysis of the dispersion relation for waves in dusty plasmas. After the choice of bi-Maxwellian distributions, and after the use of an approximation for the momentum dependent collision frequencies, the integrals which appear in the components of the dielectric tensor were written in terms of the very familiar $Z$ function, whose analytic properties are well known.

As an application of the formulation, we have considered the case of electrostatic waves propagating in the direction of the ambient magnetic field, and performed a numerical investigation comparing the magnitudes of the 'conventional' and of the 'new' contributions to the dispersion relation, for frequencies in the range of the ion-sound and Langmuir waves. The study expands previous analyses in which the case of Lang- muir waves and isotropic Maxwellian distributions has been considered [9]. To our knowledge, this is one of the first instances of numerical analysis including the effect of the 'new' contribution, which usually only appears in formal analysis of wave propagation in dusty plasmas [4, 15, 16, 19-21]. For this investigation we have considered parameters which are in the range of parameters typical of stellar winds, and the results obtained have shown that the contribution of the "new' components is very small compared to the "conventional' contribution.

The results obtained show that, for a wide range of temperature anisotropy, the 'new' contribution results in negligible effect to the dispersion relation of Langmuir and ion-sound waves, the latter also considering a wide range of the electron to ion temperature ratio.

These findings about the negligible effect of the 'new' contribution can not be generalized without further analysis to other situations, like other frequency regimes and other forms of the distribution function. For instance, the analysis of lowfrequency modes like the Alfvén wave mode would be an interesting subject for future research. We intend to proceed with the investigation of these topics in the near future. 

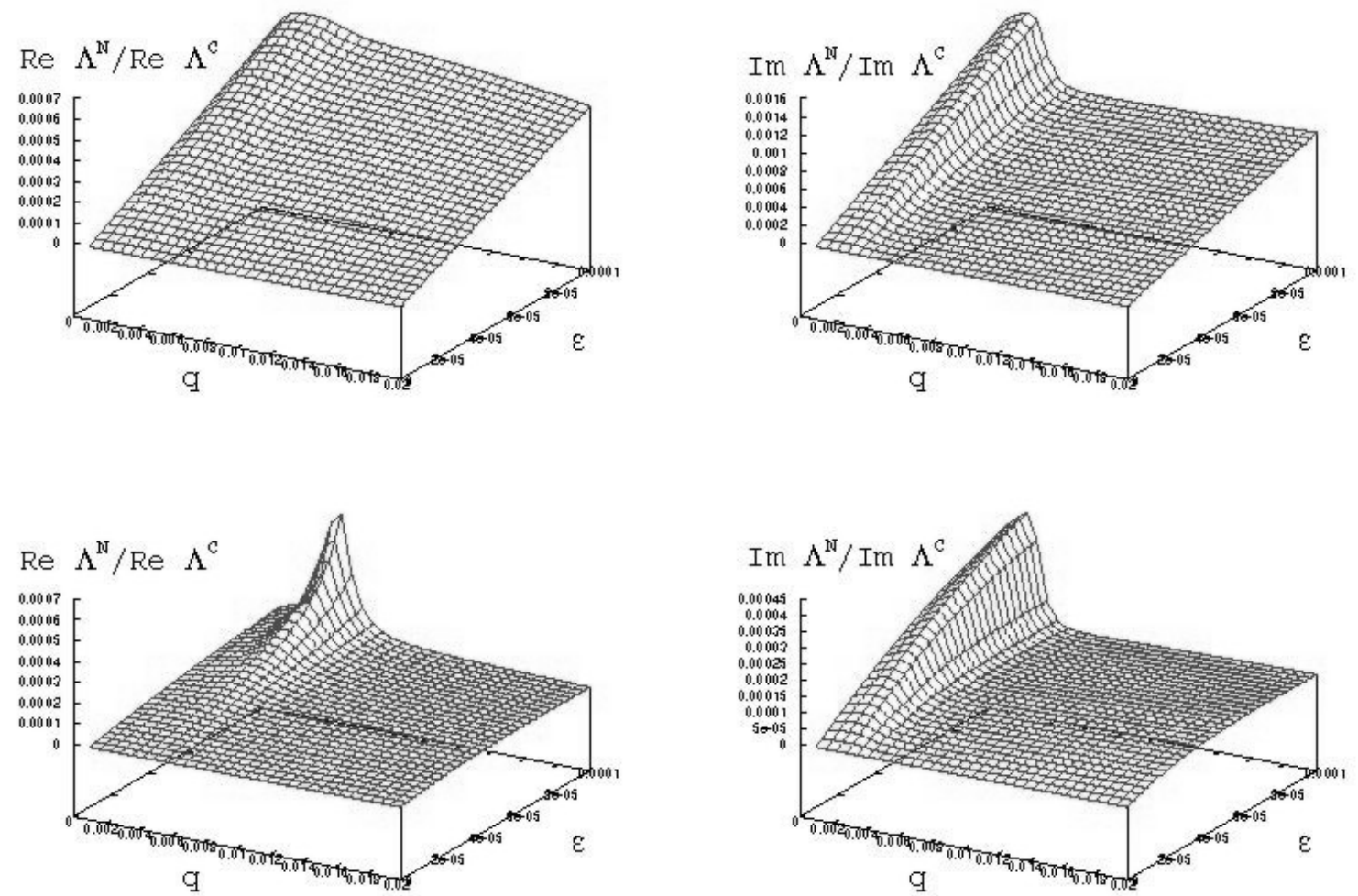

FIG. 9: Ratio between the real and imaginary parts of the 'new' and the 'conventional' contributions to the dispersion relation, vs. $q$ and $\varepsilon=n_{d} / n_{i 0}$; (upper left) ratio between the real parts $\left(\operatorname{Re} \Lambda^{N} / \operatorname{Re} \Lambda^{C}\right)$, for $\Delta_{\beta}=T_{\beta \perp} / T_{\beta \|}=0.1$, for $\beta=\mathrm{i}$,e; (upper right) ratio between the imaginary parts $\left(\operatorname{Im} \Lambda^{N} / \operatorname{Im} \Lambda^{C}\right)$, for $\Delta_{\beta}=T_{\beta \perp} / T_{\beta}=0.1$, for $\beta=\mathrm{i}$,e; (bottom left) ratio between the real parts $\left(\operatorname{Re} \Lambda^{N} / \operatorname{Re} \Lambda^{C}\right)$, for $\Delta_{\beta}=$ $T_{\beta \perp} / T_{\beta \|}=10.0$, for $\beta=\mathrm{i}$,e; (bottom right) ratio between the imaginary parts $\left(\operatorname{Im} \Lambda^{N} / \operatorname{Im} \Lambda^{C}\right.$ ), for $\Delta_{\beta}=T_{\beta \perp} / T_{\beta}=10.0$, for $\beta=\mathrm{i}, \mathrm{e} ; T_{e}=T_{i}$, and $z=\left(1.0 \times 10^{-2},-2.0 \times 10^{-4}\right)$, in the range of ion-acoustic waves. Other parameters the same as used to obtain Fig. 1 .

APPENDIX A: DETAILS OF THE EVALUATION OF INTEGRALS APPEARING IN THE EXPRESSIONS FOR THE COMPONENTS OF THE DIELECTRIC TENSOR

As we have seen, the 'conventional' contributions to the components of the dielectric tensor depend on integrals denoted as $J$ and $J_{v}$, defined by Eqs. (6) and (7). The 'new' contributions depend on integrals $J_{U}, J_{v L}, J_{v v}, J_{v 0}$ and $J_{c h}$, defined by Eqs. (12), (13), (14), (15), and (16).

In what follows, we consider the case of bi-Maxwellian distributions for ions and electrons. Moreover, as an approximation we assume that the momentum-dependent collision frequency is replaced by the average value. This approximation is adopted in order to arrive at a relatively simple estimate of the effect of the dust charge variation, effect which is frequently neglected in analysis of the dispersion relation for waves in dusty plasmas.

\section{Evaluation of the integrals $J\left(n, m, h ; f_{\beta 0}\right)$}

We start from Eq. (6),

$$
\begin{gathered}
J\left(n, m, h ; f_{\beta 0}\right) \equiv \int d^{3} u \frac{z u_{\|}^{h} u_{\perp}^{2(m-1)} u_{\perp} L\left(f_{\beta 0}\right)}{z-n r_{\beta}-q_{\|} u_{\|}+i \tilde{\vee}_{\beta d}^{0}}, \\
=\omega(2 \pi) \int_{0}^{\infty} d u_{\perp} u_{\perp} u_{\perp}^{2(m-1)} u_{\perp} \\
\times \int_{-\infty}^{\infty} d u_{\|} \frac{u_{\|}^{h} L\left(f_{\beta 0}\right)}{\omega-n \Omega_{\beta}-v_{*} k_{\|} u_{\|}+i v_{\beta d}^{0}(u)} .
\end{gathered}
$$

As we have seen, for a bi-Maxwellian distribution,

$$
L\left(f_{\beta 0}\right)=-\frac{u_{\perp}}{u_{\beta \perp}^{2}}\left[1-\frac{q_{\|}}{z} u_{\|}\left(1-\Delta_{\beta}\right)\right] f_{\beta 0} .
$$

Using this result and assuming that the collision frequency is replaced by the average value, $v_{\beta}=\int d^{3} u v_{\beta d}^{0}(u) f_{\beta 0}(u) / n_{\beta 0}$, we obtain

$J\left(n, m, h ; f_{\beta 0}\right)=-\omega \frac{n_{\beta 0}}{(2 \pi)^{1 / 2} u_{\beta \perp}^{4} u_{\beta \|}} \int_{0}^{\infty} d u_{\perp} u_{\perp}^{2 m+1} e^{-u_{\perp}^{2} /\left(2 u_{\beta \perp}^{2}\right)}$ 

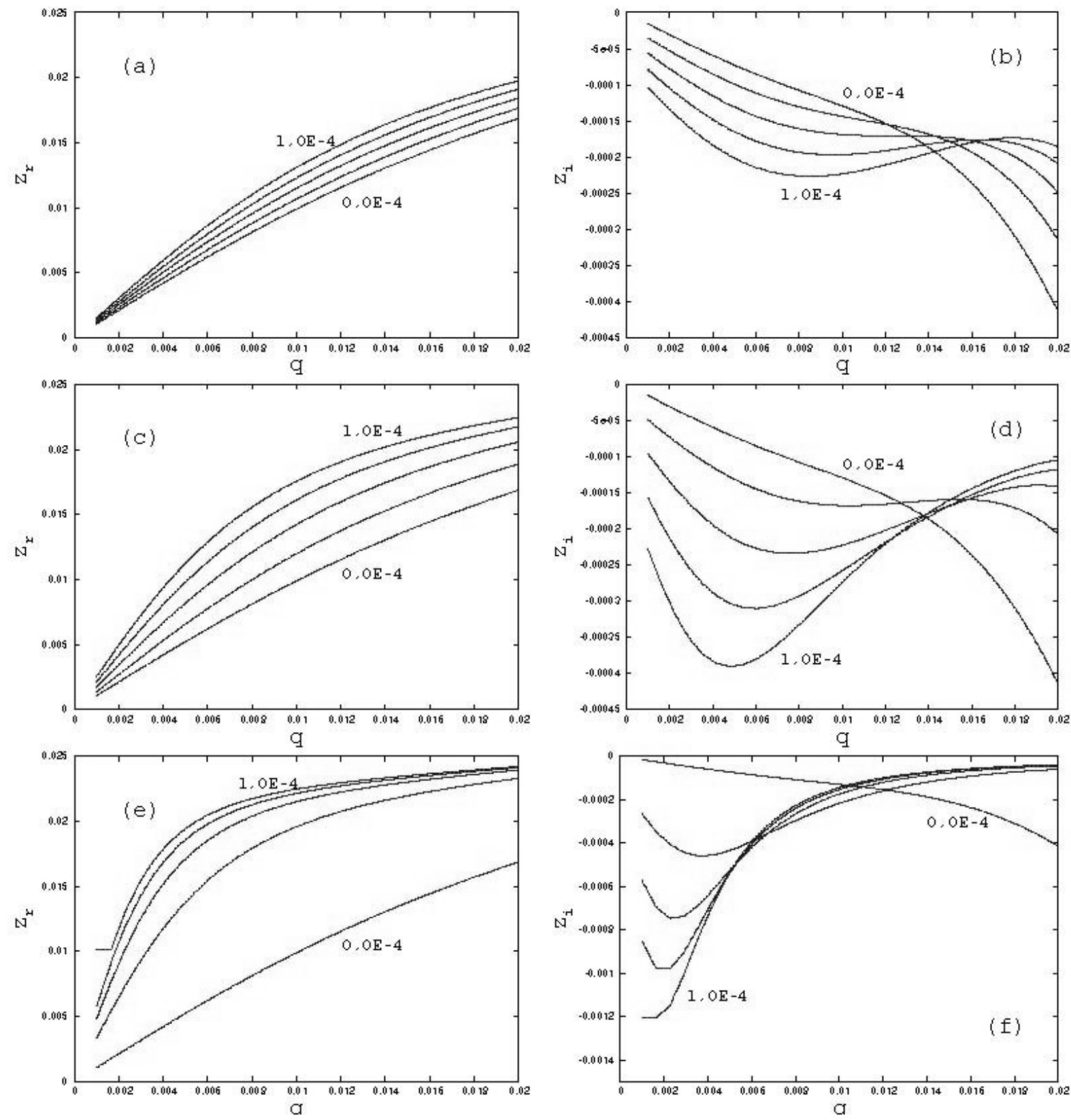

FIG. 10: Real and imaginary parts of the normalized frequency $(z)$ obtained from the dispersion relation for ion-acoustic waves, vs. $q$, for several values of $\varepsilon=n_{d} / n_{i 0}\left(0.0,2.5 \times 10^{-5}, 5.0 \times 10^{-5}, 7.5 \times 10^{-5}\right.$, and $\left.1.0 \times 10^{-4}\right)$; (a) $z_{r}$, for $T_{e \perp} / T_{e \|}=T_{i \perp} / T_{i \|}=0.1 ;(\mathrm{b}) z_{i}$, for $T_{e \perp} / T_{e \|}=$ $T_{i \perp} / T_{i \|}=0.1$; (c) $z_{r}$, for $T_{e \perp} / T_{e \|}=T_{i \perp} / T_{i \|}=1.0$; (d) $z_{i}$, for $T_{e \perp} / T_{e \|}=T_{i \perp} / T_{i \|}=1.0$; (e) $z_{r}$, for $T_{e \perp} / T_{e \|}=T_{i \perp} / T_{i \|}=10.0$; (f) $z_{i}$, for $T_{e \perp} / T_{e \|}=T_{i \perp} / T_{i \|}=10.0$; In all cases, $T_{e} / T_{i}=20.0$. Other parameters the same as used to obtain Fig. 1 .

$$
\begin{gathered}
\times \int_{-\infty}^{\infty} d u_{\|} \frac{u_{\|}^{h}\left[1-\frac{q_{\|}}{z} u_{\|}\left(1-\Delta_{\beta}\right)\right] e^{-u_{\|}^{2} /\left(2 u_{\beta \|}^{2}\right)}}{\omega-n \Omega_{\beta}-v_{*} k_{\|} u_{\|}+i v_{\beta}} \\
=\frac{\omega}{v_{*} k_{\|}} \frac{2^{m}(m !)}{(2 \pi)^{1 / 2}} \frac{n_{\beta 0}}{u_{\beta \|}} u_{\beta \perp}^{2(m-1)} \\
\times \int_{-\infty}^{\infty} d u_{\|} \frac{u_{\|}^{h}\left[1-\frac{q_{\|}}{z} u_{\|}\left(1-\Delta_{\beta}\right)\right] e^{-u_{\|}^{2} /\left(2 u_{\beta \|}^{2}\right)}}{u_{\|}-u_{\|, \text {res }}},
\end{gathered}
$$

where

$$
u_{\|, \text {res }}=\frac{\omega-n \Omega_{\beta}+i v_{\beta}}{v_{*} k_{\|}} .
$$

Introducing a change of variables such that $u_{\|}=\sqrt{2} u_{\beta \|} t$,

$$
\begin{gathered}
J\left(n, m, h ; f_{\beta 0}\right)=\frac{\omega}{v_{*} k_{\|}} \frac{2^{m}(m !)}{(2 \pi)^{1 / 2}} \frac{n_{\beta 0}}{u_{\beta \|}} u_{\beta \perp}^{2(m-1)}\left(\sqrt{2} u_{\beta \|}\right)^{h} \\
\times\left(\int_{-\infty}^{\infty} d t \frac{t^{h} e^{-t^{2}}}{t-t_{\|, r e s}}-\frac{q_{\|}}{z}\left(1-\Delta_{\beta}\right) \sqrt{2} u_{\beta \|} \int_{-\infty}^{\infty} d t \frac{t^{h+1} e^{-t^{2}}}{t-\hat{\zeta}_{\beta}^{n}}\right),
\end{gathered}
$$



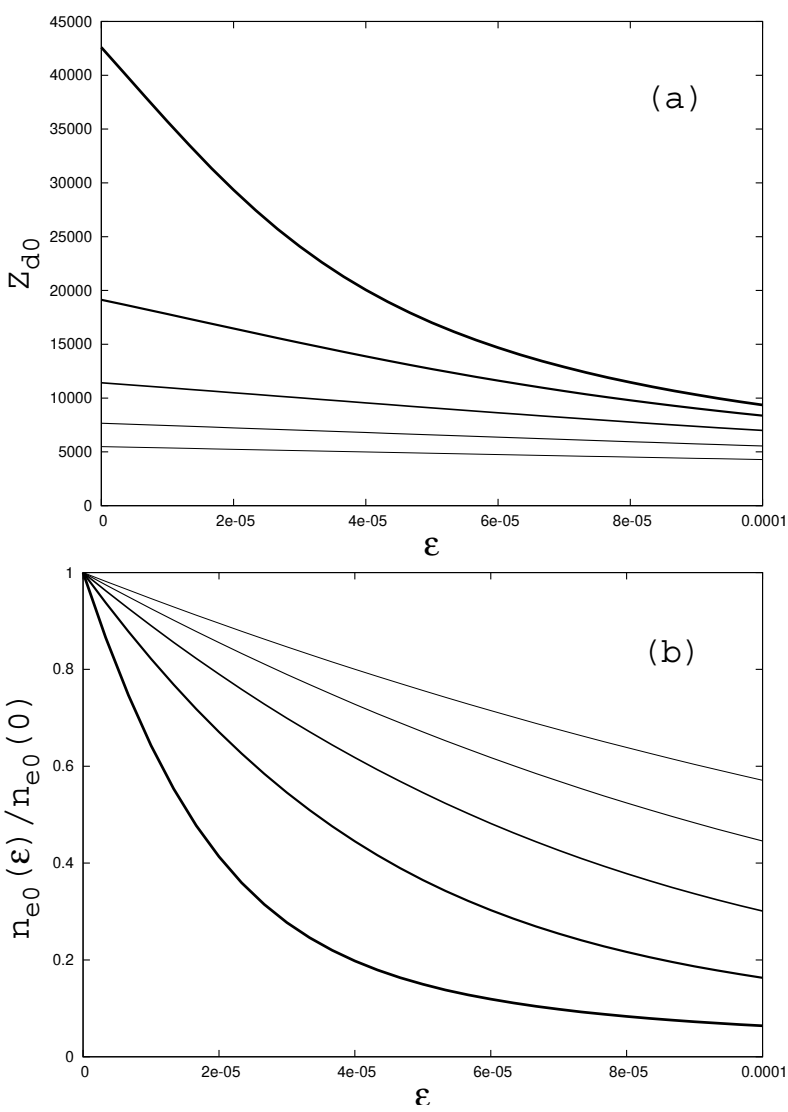

FIG. 11: (a) $Z_{d 0}$ as a function of $\varepsilon$ for the case of ion-sound waves, for five values of the ratio $T_{\perp} / T_{\|}$, (b) $n_{e 0}$ as a function of $\varepsilon$ for the case of ion-sound waves, for five values of the ratio $T_{\perp} / T_{\|}$. For ionsound waves, it is assumed that $T_{e \|} / T_{i \|}=10.0$. From the thinnest to the thickest, the curves show the cases of $T_{\perp} / T_{\|}=0.2,0.5,1.0,2.0$, and 5.0, for other parameters as in Fig. 1.

where

$$
\hat{\zeta}_{\beta}^{n}=\frac{z-n r_{\beta}+i \tilde{v}_{\beta}}{\sqrt{2} q_{\|} u_{\beta \|}}
$$

Re-arranging the expression, we obtain,

$$
\begin{gathered}
J\left(n, m, h ; f_{\beta 0}\right)=n_{\beta 0}(m !)(\sqrt{2})^{2 m+h} u_{\beta \perp}^{2(m-1)} u_{\beta \|}^{h} \frac{1}{\sqrt{\pi}} \\
\times\left(\zeta_{\beta}^{0} \int_{-\infty}^{\infty} d t \frac{t^{h} e^{-t^{2}}}{t-\hat{\zeta}_{\beta}^{n}}-\left(1-\Delta_{\beta}\right) \int_{-\infty}^{\infty} d t \frac{t^{h+1} e^{-t^{2}}}{t-\hat{\zeta}_{\beta}^{n}}\right),
\end{gathered}
$$

where $\zeta_{\beta}^{0}=z /\left(\sqrt{2} q_{\|} u_{\beta}\right)$. We note that the integral over $u_{\perp}$ was made assuming that $m$ is integer.

In order to proceed, we consider the integral appearing in Eq. (A1), which depend on an integer power of $t, t^{\ell}$.

For $\ell=0$,

$$
\frac{1}{\sqrt{\pi}} \int_{-\infty}^{\infty} d t \frac{e^{-t^{2}}}{t-\hat{\zeta}_{\beta}^{n}}=Z\left(\hat{\zeta}_{\beta}^{n}\right) .
$$
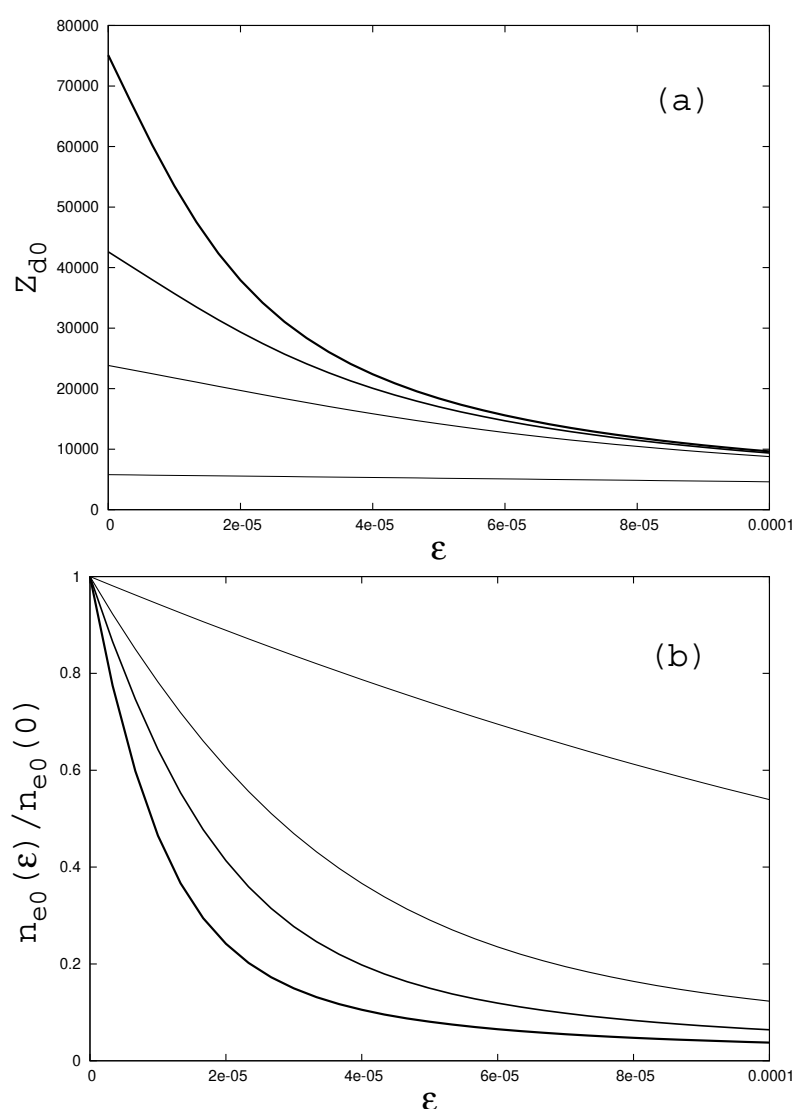

FIG. 12: (a) $Z_{d 0}$ as a function of $\varepsilon$ for the case of ion-sound waves, for four values of the ratio $T_{e \|} / T_{i \|}$, with $T_{\perp} / T_{\|}=5.0$. (b) $n_{e 0}$ as a function of $\varepsilon$ for the case of ion-sound waves, for four values of the ratio $T_{e \|} / T_{i \|}$, with $T_{\perp} / T_{\|}=5.0$. From the thinnest to the thickest, the curves show the cases of $T_{e \|} / T_{i \|}=1.0,5.0,10.0$, and 20.0, with other parameters as in Fig. 1.

For $\ell=1$,

$$
\begin{gathered}
\frac{1}{\sqrt{\pi}} \int_{-\infty}^{\infty} d t \frac{t e^{-t^{2}}}{t-\hat{\zeta}_{\beta}^{n}}=\frac{1}{\sqrt{\pi}} \int_{-\infty}^{\infty} d t \frac{\left(t-\hat{\zeta}_{\beta}^{n}+\hat{\zeta}_{\beta}^{n}\right) e^{-t^{2}}}{t-\hat{\zeta}_{\beta}^{n}} \\
=\frac{1}{\sqrt{\pi}}\left[\int_{-\infty}^{\infty} d t e^{-t^{2}}+\hat{\zeta}_{\beta}^{n} \int_{-\infty}^{\infty} d t \frac{e^{-t^{2}}}{t-\hat{\zeta}_{\beta}^{n}}\right] \\
=1+\hat{\zeta}_{\beta}^{n} Z\left(\hat{\zeta}_{\beta}^{n}\right) .
\end{gathered}
$$

For $\ell=2$,

$$
\begin{gathered}
\frac{1}{\sqrt{\pi}} \int_{-\infty}^{\infty} d t \frac{t^{2} e^{-t^{2}}}{t-\hat{\zeta}_{\beta}^{n}}=\frac{1}{\sqrt{\pi}} \int_{-\infty}^{\infty} d t \frac{t\left(t-\hat{\zeta}_{\beta}^{n}+\hat{\zeta}_{\beta}^{n}\right) e^{-t^{2}}}{t-\hat{\zeta}_{\beta}^{n}} \\
=\frac{1}{\sqrt{\pi}}\left[\int_{-\infty}^{\infty} d t t e^{-t^{2}}+\hat{\zeta}_{\beta}^{n} \int_{-\infty}^{\infty} d t \frac{t e^{-t^{2}}}{t-\hat{\zeta}_{\beta}^{n}}\right] \\
=\hat{\zeta}_{\beta}^{n}\left[1+\hat{\zeta}_{\beta}^{n} Z\left(\hat{\zeta}_{\beta}^{n}\right)\right] .
\end{gathered}
$$



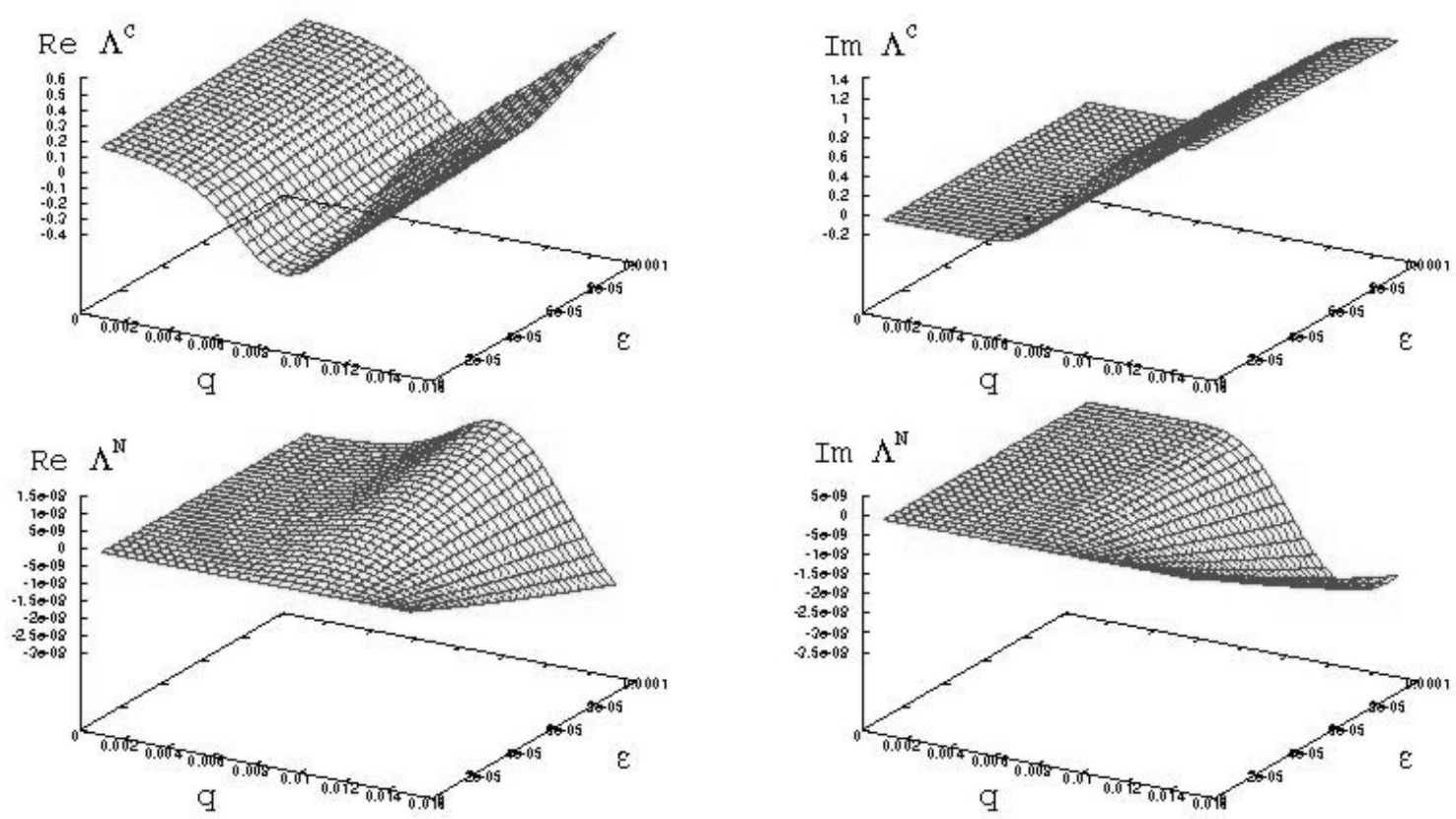

FIG. 13: (upper left) Real part of the 'conventional' contribution to the dispersion relation, vs. $q$ and $\varepsilon=n_{d} / n_{i 0}$; (upper right) imaginary part of the 'conventional' contribution; (bottom left) Real part of the 'new' contribution; (bottom right) imaginary part of the 'new' contribution; $T_{e}=T_{i}, \Delta_{\beta}=T_{\beta \perp} / T_{\beta \|}=0.1$, for $\beta=i, e$, and $z=\left(1.1 \times 10^{0},-1.0 \times 10^{-3}\right)$, in the range of Langmuir waves. $T_{i}=1.0 \times 10^{4} \mathrm{~K}, n_{i 0}=1.0 \times 10^{9}$ $\mathrm{cm}^{-3}, Z_{i}=1.0, m_{i}=m_{p}$, where $m_{p}$ is the proton mass, and $a=1.0 \times 10^{-4}$.

For $\ell=3$,

$$
\begin{gathered}
\frac{1}{\sqrt{\pi}} \int_{-\infty}^{\infty} d t \frac{t^{3} e^{-t^{2}}}{t-\hat{\zeta}_{\beta}^{n}}=\frac{1}{\sqrt{\pi}} \int_{-\infty}^{\infty} d t \frac{t^{2}\left(t-\hat{\zeta}_{\beta}^{n}+\hat{\zeta}_{\beta}^{n}\right) e^{-t^{2}}}{t-\hat{\zeta}_{\beta}^{n}} \\
=\frac{1}{\sqrt{\pi}}\left[\int_{-\infty}^{\infty} d t t^{2} e^{-t^{2}}+\hat{\zeta}_{\beta}^{n} \int_{-\infty}^{\infty} d t \frac{t^{2} e^{-t^{2}}}{t-\hat{\zeta}_{\beta}^{n}}\right] \\
=\left\{\frac{1}{2}+\left(\hat{\zeta}_{\beta}^{n}\right)^{2}\left[1+\hat{\zeta}_{\beta}^{n} Z\left(\hat{\zeta}_{\beta}^{n}\right)\right]\right\} .
\end{gathered}
$$

The result is (for integer $m$ ),

$$
\begin{gathered}
J\left(n, m, 0 ; f_{\beta 0}\right)=(m !)(\sqrt{2})^{2 m} n_{\beta 0} u_{\beta \perp}^{2(m-1)} \\
\times\left\{\zeta_{\beta}^{0} Z\left(\hat{\zeta}_{\beta}^{n}\right)-\left(1-\Delta_{\beta}\right)\left[1+\hat{\zeta}_{\beta}^{n} Z\left(\hat{\zeta}_{\beta}^{n}\right)\right]\right\}, \\
J\left(n, m, 1 ; f_{\beta 0}\right)=(m !)(\sqrt{2})^{2 m+1} n_{\beta 0} u_{\beta \perp}^{2(m-1)} u_{\beta} \| \\
\times\left\{\zeta_{\beta}^{0}\left[1+\hat{\zeta}_{\beta}^{n} Z\left(\hat{\zeta}_{\beta}^{n}\right)\right]-\left(1-\Delta_{\beta}\right) \hat{\zeta}_{\beta}^{n}\left[1+\hat{\zeta}_{\beta}^{n} Z\left(\hat{\zeta}_{\beta}^{n}\right)\right]\right\}, \\
J\left(n, m, 2 ; f_{\beta 0}\right)=(m !)(\sqrt{2})^{2 m+2} n_{\beta 0} u_{\beta \perp}^{2(m-1)} u_{\beta \|}^{2}
\end{gathered}
$$$$
\times\left\{\zeta_{\beta}^{0} \hat{\zeta}_{\beta}^{n}\left[1+\hat{\zeta}_{\beta}^{n} Z\left(\hat{\zeta}_{\beta}^{n}\right)\right]-\left(1-\Delta_{\beta}\right)\left\{\frac{1}{2}+\left(\hat{\zeta}_{\beta}^{n}\right)^{2}\left[1+\hat{\zeta}_{\beta}^{n} Z\left(\hat{\zeta}_{\beta}^{n}\right)\right]\right\}\right\}
$$

\section{Evaluation of the integrals $J_{v}\left(n, m, h ; f_{\beta 0}\right)$}

We start from Eq. (7),

$$
J_{v}\left(n, m, h ; f_{\beta 0}\right)=\int d^{3} u\left[\frac{v_{\beta d}^{0}(u)}{\Omega_{*}}\right] \frac{u_{\|}^{h} u_{\perp}^{2(m-1)} u_{\perp} \mathcal{L}\left(f_{\beta 0}\right)}{z-n r_{\beta}-q_{\|} u_{\|}+i \tilde{v}_{\beta d}^{0}} .
$$

Using the average value of the collision frequency, considering a bi-Maxwellian distribution, and using Eq. (18),

$$
\begin{aligned}
& J_{v}\left(n, m, h ; f_{\beta 0}\right)=-\frac{\tilde{\mathrm{v}}_{\beta} n_{\beta 0}\left(1-\Delta_{\beta}\right)}{(2 \pi)^{1 / 2} u_{\beta \perp}^{4} u_{\beta} \|} \int_{0}^{\infty} d u_{\perp} u_{\perp}^{2 m+1} e^{-u_{\perp}^{2} /\left(2 u_{\beta \perp}^{2}\right)} \\
& \times \int_{-\infty}^{\infty} d u_{\|} \frac{u_{\|}^{h+1} e^{-u_{\|}^{2} /\left(2 u_{\beta \|}^{2}\right)}}{z-n r_{\beta}-q_{\|} u_{\|}+i \tilde{v}_{\beta}} \\
&=-\frac{\tilde{\mathrm{v}}_{\beta} n_{\beta 0}\left(1-\Delta_{\beta}\right)}{(2 \pi)^{1 / 2} u_{\beta \perp}^{4} u_{\beta \|}} \frac{m !}{2}\left(2 u_{\beta \perp}^{2}\right)^{m+1} \\
& \times \int_{-\infty}^{\infty} d u_{\|} \frac{u_{\|}^{h+1} e^{-u_{\|}^{2} /\left(2 u_{\beta \|}^{2}\right)}}{z-n r_{\beta}-q_{\|} u_{\|}+i \tilde{\mathrm{v}}_{\beta}} \cdot \\
&=(\sqrt{2})^{2 m+h}(m !) \frac{\tilde{\mathrm{v}}_{\beta}}{q_{\|}} n_{\beta 0}\left(1-\Delta_{\beta}\right) u_{\beta \perp}^{2(m-1)} u_{\beta \|}^{h}
\end{aligned}
$$



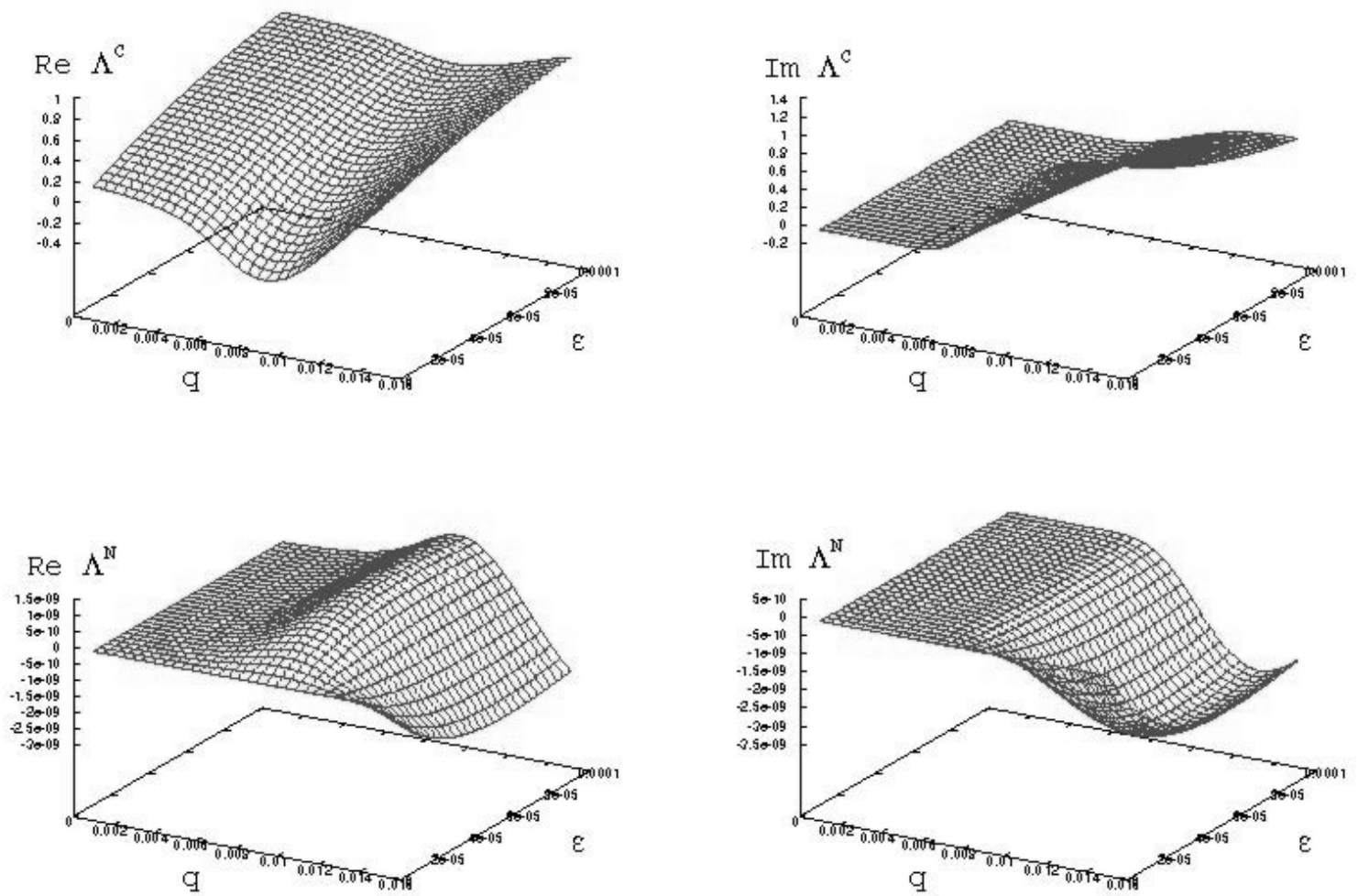

FIG. 14: (upper left) Real part of the 'conventional' contribution to the dispersion relation, vs. $q$ and $\varepsilon=n_{d} / n_{i 0}$; (upper right) imaginary part of the 'conventional' contribution; (bottom left) Real part of the 'new' contribution; (bottom right) imaginary part of the 'new' contribution; $T_{e}=T_{i}, \Delta_{\beta}=T_{\beta \perp} / T_{\beta \|}=10.0$, for $\beta=i, e$, and $z=\left(1.1 \times 10^{0},-1.0 \times 10^{-3}\right)$, in the range of Langmuir waves. Other parameters the same as used to obtain Fig. 13 .

$$
\times \frac{1}{\sqrt{\pi}} \int_{-\infty}^{\infty} d t \frac{t^{h+1} e^{-t^{2}}}{t-\hat{\zeta}_{\beta}^{n}} .
$$

The result is, for some values of $h$,

$$
\begin{gathered}
J_{v}\left(n, m, 0 ; f_{\beta 0}\right)=(m !)(\sqrt{2})^{2 m}\left(1-\Delta_{\beta}\right) n_{\beta 0} \\
\times u_{\beta \perp}^{2(m-1)} \frac{\tilde{v}_{\beta}}{q_{\|}}\left[1+\hat{\zeta}_{\beta}^{n} Z\left(\hat{\zeta}_{\beta}^{n}\right)\right], \\
J_{v}\left(n, m, 1 ; f_{\beta 0}\right)=(m !)(\sqrt{2})^{2 m+1}\left(1-\Delta_{\beta}\right) n_{\beta 0} \\
\times u_{\beta \perp}^{2(m-1)} u_{\beta \|} \frac{\tilde{v}_{\beta}}{q_{\|}} \hat{\zeta}_{\beta}^{n}\left[1+\hat{\zeta}_{\beta}^{n} Z\left(\hat{\zeta}_{\beta}^{n}\right)\right], \\
J_{v}\left(n, m, 2 ; f_{\beta 0}\right)=(m !)(\sqrt{2})^{2 m+2}\left(1-\Delta_{\beta}\right) n_{\beta 0} \\
\times u_{\beta \perp}^{2(m-1)} u_{\beta \|}^{2} \frac{\tilde{v}_{\beta}}{q_{\|}}\left\{\frac{1}{2}+\left(\hat{\zeta}_{\beta}^{n}\right)^{2}\left[1+\hat{\zeta}_{\beta}^{n} Z\left(\hat{\zeta}_{\beta}^{n}\right)\right]\right\} .
\end{gathered}
$$

\section{Evaluation of $e_{z z}$}

From Eq. (5), we see that $e_{z z}$ features three terms. Two of these terms can be evaluated with the use of the $J$ and $J_{v}$ integrals. The other term will be evaluated here, for a biMaxwellian distribution. We write

$$
\begin{gathered}
e_{z z}=e_{z z}^{1}+\frac{1}{z^{2}} \sum_{\beta} \frac{\omega_{p \beta}^{2}}{\Omega_{*}^{2}} \frac{1}{n_{\beta 0}} a(0,0)\left[J\left(0,0,2 ; f_{\beta 0}\right)+i J_{v}\left(0,0,1 ; f_{\beta 0}\right)\right], \\
e_{z z}^{1}=-\frac{1}{z^{2}} \sum_{\beta} \frac{\omega_{p \beta}^{2}}{\Omega_{*}^{2}} \frac{1}{n_{\beta 0}} \int d^{3} u \frac{u_{\|}}{u_{\perp}} \mathcal{L}\left(f_{\beta 0}\right) .
\end{gathered}
$$

Using Eq. (18), the $e_{z z}^{1}$ term can be written as

$$
\begin{gathered}
e_{z z}^{1}=\frac{1}{z^{2}} \sum_{\beta} \frac{\omega_{p \beta}^{2}}{\Omega_{*}^{2}} \frac{\left(1-\Delta_{\beta}\right)}{(2 \pi)^{1 / 2} u_{\beta \perp}^{4} u_{\beta \|}} \\
\times \int_{0}^{\infty} d u_{\perp} u_{\perp} e^{-u_{\perp}^{2} /\left(2 u_{\beta \perp}^{2}\right)} \int_{-\infty}^{\infty} d u_{\|} u_{\|}^{2} e^{-u_{\|}^{2} /\left(2 u_{\beta \|}^{2}\right)} \\
=\frac{1}{z^{2}} \sum_{\beta} \frac{\omega_{p \beta}^{2}}{\Omega_{*}^{2}} \frac{\left(1-\Delta_{\beta}\right)}{(2 \pi)^{1 / 2} u_{\beta \perp}^{4} u_{\beta \|}}\left(\frac{2 u_{\beta \perp}^{2}}{2}\right)\left(\frac{\sqrt{\pi}}{2}\left(2 u_{\beta \|}^{2}\right)^{3 / 2}\right)
\end{gathered}
$$



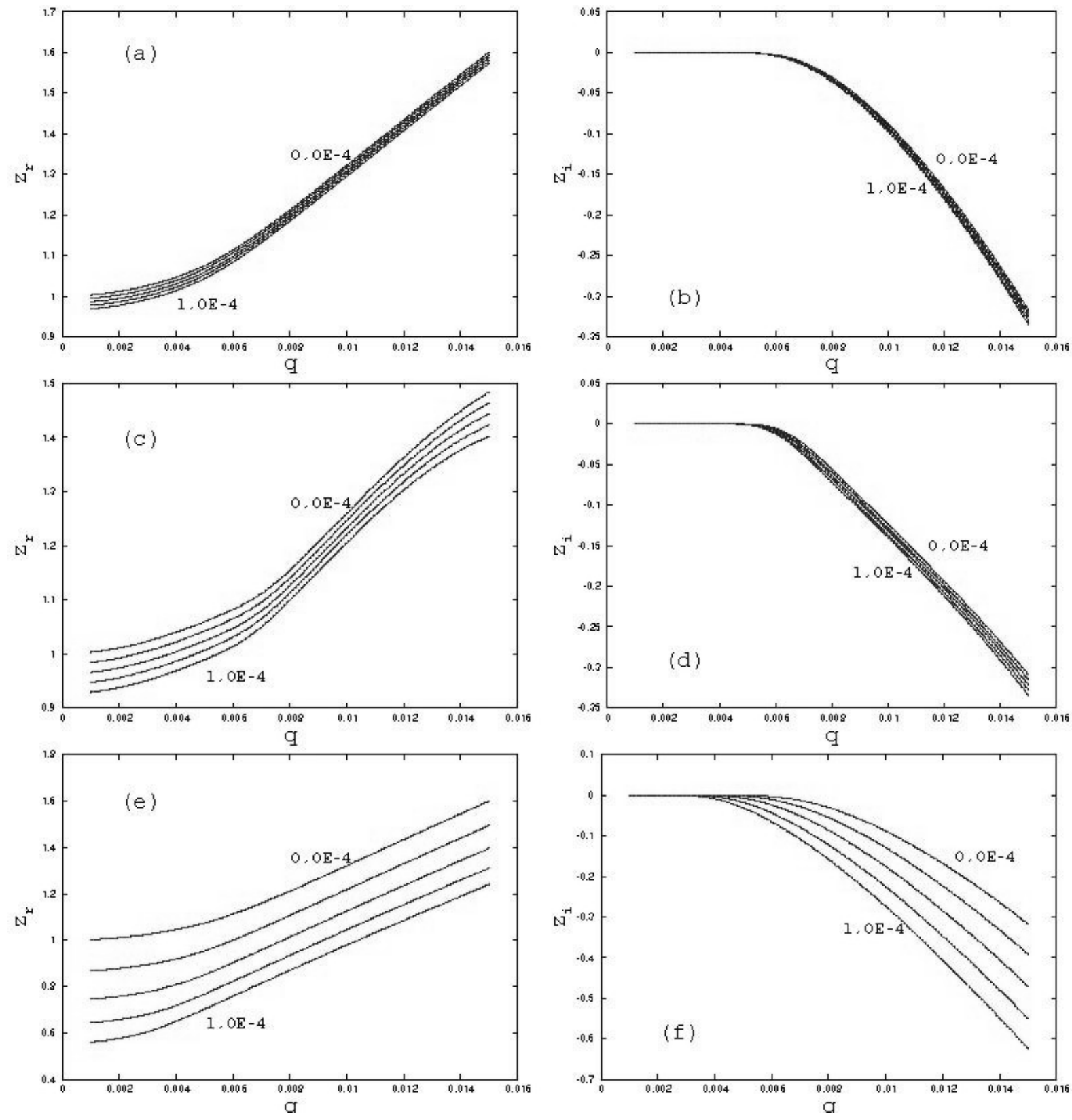

FIG. 15: Real and imaginary parts of the normalized frequency $(z)$ obtained from the dispersion relation for Langmuir waves, vs. $q$, for several values of $\varepsilon=n_{d} / n_{i 0}\left(0.0,2.5 \times 10^{-5}, 5.0 \times 10^{-5}, 7.5 \times 10^{-5}\right.$, and $\left.1.0 \times 10^{-4}\right) ;$ (a) $z_{r}$, for $T_{e \perp} / T_{e \|}=T_{i \perp} / T_{i \|}=0.1$; (b) $z_{i}$, for $T_{e \perp} / T_{e \|}=$ $T_{i \perp} / T_{i \|}=0.1$; (c) $z_{r}$, for $T_{e \perp} / T_{e \|}=T_{i \perp} / T_{i \|}=1.0$; (d) $z_{i}$, for $T_{e \perp} / T_{e \|}=T_{i \perp} / T_{i \|}=1.0$; (e) $z_{r}$, for $T_{e \perp} / T_{e \|}=T_{i \perp} / T_{i \|}=10.0$; (f) $z_{i}$, for $T_{e \perp} / T_{e \|}=T_{i \perp} / T_{i \|}=10.0$; In all cases, $T_{e}=T_{i}$. Other parameters the same as used to obtain Fig. 13.

$$
=\frac{1}{z^{2}} \sum_{\beta} \frac{\omega_{p \beta}^{2}}{\Omega_{*}^{2}} \frac{u_{\beta \|}^{2}}{u_{\beta \perp}^{2}}\left(1-\Delta_{\beta}\right)=\frac{1}{z^{2}} \sum_{\beta} \frac{\omega_{p \beta}^{2}}{\Omega_{*}^{2}} \frac{\left(1-\Delta_{\beta}\right)}{\Delta_{\beta}} .
$$

Therefore,

$$
\begin{aligned}
e_{z z} & =\frac{1}{z^{2}} \sum_{\beta} \frac{\omega_{p \beta}^{2}}{\Omega_{*}^{2}} \frac{\left(1-\Delta_{\beta}\right)}{\Delta_{\beta}}+\frac{1}{z^{2}} \sum_{\beta} \frac{\omega_{p \beta}^{2}}{\Omega_{*}^{2}} \frac{1}{n_{\beta 0}} \\
& \times\left[J\left(0,0,2 ; f_{\beta 0}\right)+i J_{v}\left(0,0,1 ; f_{\beta 0}\right)\right] .
\end{aligned}
$$

\section{Evaluation of the integrals $J_{U}\left(n, m, h, l ; f_{\beta 0}\right)$}

We start from Eq. (12),

$$
\begin{gathered}
J_{U}\left(n, m, h, l ; f_{\beta 0}\right)=z \int d^{3} u\left(\frac{\tilde{\mathrm{v}}_{\beta d}^{0}}{z}\right)^{l} \frac{f_{\beta 0}}{z-n r_{\beta}-q_{\|} u_{\|}+i \tilde{\mathrm{v}}_{\beta d}^{0}} \\
\times \frac{u_{\|}^{h} u_{\perp}^{2 m}}{u} H\left(u^{2}+\frac{2 Z_{d 0} e q_{\beta}}{a m_{\beta} v_{*}^{2}}\right) .
\end{gathered}
$$

We assume, for simplicity, that the collision frequency is 

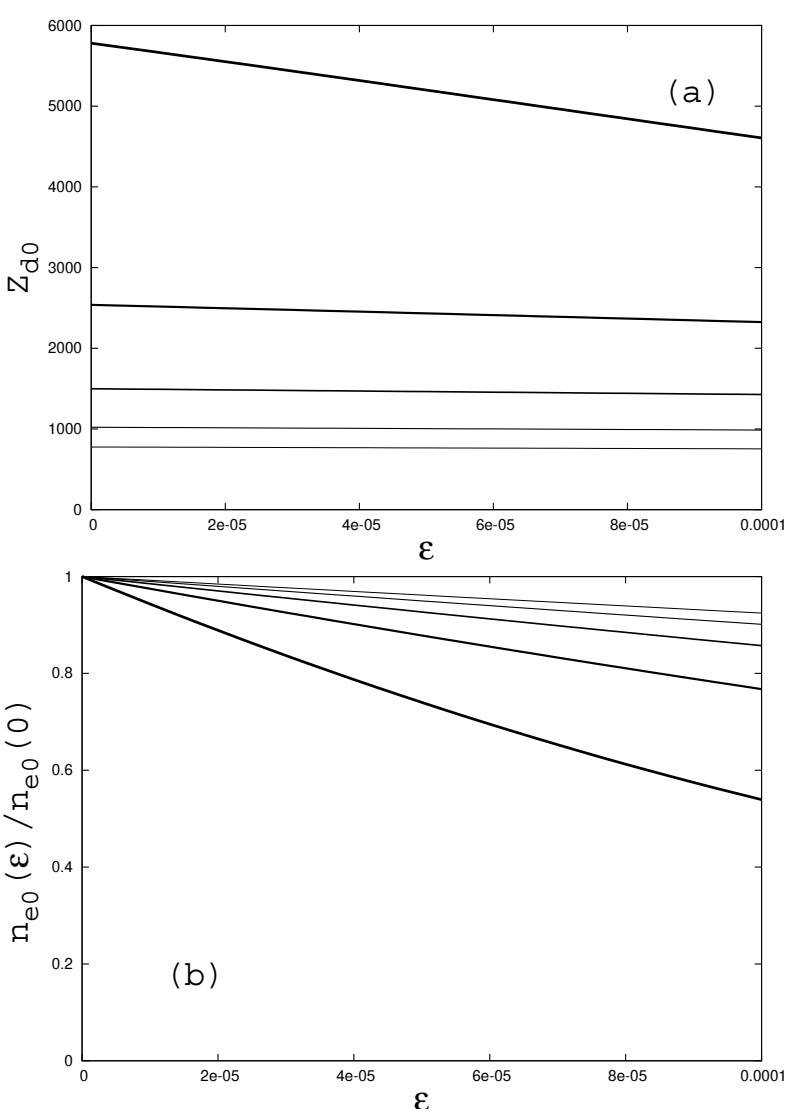

FIG. 16: (a) $Z_{d 0}$ as a function of $\varepsilon$ for the case of Langmuir waves, for five values of the ratio $T_{\perp} / T_{\|}$. (b) $n_{e 0}$ as a function of $\varepsilon$ for the case of Langmuir waves, for five values of the ratio $T_{\perp} / T_{\|}$. For Langmuir waves, it is assumed that ion and electron temperatures are equal. From the thinnest to the thickest, the curves show the cases of $T_{\perp} / T_{\|}=0.2,0.5,1.0,2.0$, and 5.0, with other parameters as in Fig. 13.

replaced by the average value, and also neglect the effect of the Heaviside function in the numerator of the integrand. This approximation can be understood as follows: The collision frequency (for electrons) already contains a step function, which therefore don't need to be written explicitly in the integrand. Afterwards, we replace the collision frequency by the average value, and obtain

$J_{U}\left(n, m, h, l ; f_{\beta 0}\right)=z\left(\frac{\tilde{\mathrm{v}}_{\beta}}{z}\right)^{l} \int d^{3} u \frac{f_{\beta 0}}{z-n r_{\beta}-q_{\|} u_{\|}+i \tilde{\mathrm{v}}_{\beta}} \frac{u_{\|}^{h} u_{\perp}^{2 m}}{u}$.

We further approximate, by using $u \simeq u_{\perp}$. For a biMaxwellian distribution, we therefore obtain

$$
\begin{gathered}
J_{U}\left(n, m, h, l ; f_{\beta 0}\right)=-\left(\frac{\tilde{\mathrm{v}}_{\beta}}{z}\right)^{l} \frac{z}{q_{\|}} \frac{n_{\beta 0}}{(2 \pi)^{1 / 2} u_{\beta \perp}^{2} u_{\beta \|}} \\
\times\left(\sqrt{2} u_{\beta \|}\right)^{h} \int_{0}^{\infty} d u_{\perp} u_{\perp}^{2 m} e^{-u_{\perp}^{2} /\left(2 u_{\beta \perp}^{2}\right)} \int_{-\infty}^{\infty} d t \frac{t^{h} e^{-t^{2}}}{t-\hat{\zeta}_{\beta}^{n}}, \\
=-\left(\frac{\tilde{\mathrm{v}}_{\beta}}{z}\right)^{l} \frac{z}{\sqrt{2} q_{\|} u_{\beta \|}} \frac{n_{\beta 0}}{u_{\beta \perp}^{2}} \frac{\left(2 u_{\beta \perp}^{2}\right)^{m+1 / 2}}{2}
\end{gathered}
$$

$$
\begin{gathered}
\times \Gamma\left(m+\frac{1}{2}\right)\left(\sqrt{2} u_{\beta \|}\right)^{h} \frac{1}{\sqrt{\pi}} \int_{-\infty}^{\infty} d t \frac{t^{h} e^{-t^{2}}}{t-\hat{\zeta}_{\beta}^{n}}, \\
J_{U}\left(n, m, h, l ; f_{\beta 0}\right)=-\Gamma\left(m+\frac{1}{2}\right)(\sqrt{2})^{2 m-1+h}\left(\frac{\tilde{\mathrm{v}}_{\beta}}{z}\right)^{l} n_{\beta 0} \\
\times u_{\beta \perp}^{2 m-1} u_{\beta \|}^{h} \zeta_{\beta}^{0} \frac{1}{\sqrt{\pi}} \int_{-\infty}^{\infty} d t \frac{t^{h} e^{-t^{2}}}{t-\hat{\zeta}_{\beta}^{n}} .
\end{gathered}
$$

The result is, for some values of $h$,

$$
\begin{gathered}
J_{U}\left(n, m, 0, l ; f_{\beta 0}\right) \simeq-\Gamma\left(m+\frac{1}{2}\right)(\sqrt{2})^{2 m-1+h}\left(\frac{\tilde{\mathrm{v}}_{\beta}}{z}\right)^{l} \\
\times n_{\beta 0} u_{\beta \perp}^{2 m-1} u_{\beta \|}^{h} \zeta_{\beta}^{0} Z\left(\hat{\zeta}_{\beta}^{n}\right), \\
J_{U}\left(n, m, 1, l ; f_{\beta 0}\right) \simeq-\Gamma\left(m+\frac{1}{2}\right)(\sqrt{2})^{2 m-1+h}\left(\frac{\tilde{\mathrm{v}}_{\beta}}{z}\right)^{l} \\
\times n_{\beta 0} u_{\beta \perp}^{2 m-1} u_{\beta \|}^{h} \zeta_{\beta}^{0}\left[1+\hat{\zeta}_{\beta}^{n} Z\left(\hat{\zeta}_{\beta}^{n}\right)\right], \\
J_{U}\left(n, m, 2, l ; f_{\beta 0}\right) \simeq-\Gamma\left(m+\frac{1}{2}\right)(\sqrt{2})^{2 m-1+h}\left(\frac{\tilde{\mathrm{v}}_{\beta}}{z}\right)^{l} \\
\times n_{\beta 0} u_{\beta \perp}^{2 m-1} u_{\beta \|}^{h} \zeta_{\beta}^{0} \hat{\zeta}_{\beta}^{n}\left[1+\hat{\zeta}_{\beta}^{n} Z\left(\hat{\zeta}_{\beta}^{n}\right)\right] .
\end{gathered}
$$

\section{Evaluation of the integrals $J_{v L}\left(n, m, h ; f_{\beta 0}\right)$}

We start from Eq. (13),

$$
J_{v L}\left(n, m, h ; f_{\beta 0}\right)=z \int d^{3} u \frac{\tilde{\mathrm{v}}_{\beta d}^{0}}{z} \frac{u_{\|}^{h} u_{\perp}^{2 m-1} L\left(f_{\beta 0}\right)}{z-n r_{\beta}-q_{\|} u_{\|}+i \tilde{v}_{\beta d}^{0}} .
$$

Using the average value of the collision frequency, we obtain

$$
J_{v L}\left(n, m, h ; f_{\beta 0}\right)=\frac{\tilde{v}_{\beta}}{z} z \int d^{3} u \frac{u_{\|}^{h} u_{\perp}^{2(m-1)} u_{\perp} L\left(f_{\beta 0}\right)}{z-n r_{\beta}-q_{\|} u_{\|}+i \tilde{v}_{\beta}}
$$

which, by comparison with Eq. (6), can be written as follows,

$$
J_{v L}\left(n, m, h ; f_{\beta 0}\right)=\frac{\tilde{\mathrm{v}}_{\beta}}{z} J\left(n, m, h ; f_{\beta 0}\right)
$$

where the $J\left(n, m, h ; f_{\beta 0}\right)$ are given by Eqs. (A1) and (A6). 


\section{Evaluation of the integrals $J_{v v}\left(n, m ; f_{\beta 0}\right)$}

We start from Eq. (14),

$$
J_{v v}\left(n, m ; f_{\beta 0}\right)=z \int d^{3} u\left(\frac{\tilde{\mathrm{v}}_{\beta d}^{0}}{z}\right)^{2} \frac{u_{\perp}^{2 m-1} \mathcal{L}\left(f_{\beta 0}\right)}{z-n r_{\beta}-q_{\|} u_{\|}+i \tilde{\mathrm{v}}_{\beta d}^{0}} .
$$

Considering a bi-Maxwellian distribution, using Eq. (18), and using the average value of the collision frequency, we obtain

$$
\begin{gathered}
J_{v v}\left(n, m ; f_{\beta 0}\right)=-\left(\frac{\tilde{\mathrm{v}}_{\beta}}{z}\right)^{2} z \frac{\left(1-\Delta_{\beta}\right) n_{\beta 0}}{(2 \pi)^{1 / 2} u_{\beta \perp}^{2} u_{\beta \|}} \\
\times \int_{0}^{\infty} d u_{\perp} u_{\perp} u_{\perp}^{2 m-1} \frac{u_{\perp}}{u_{\beta \perp}^{2}} e^{-u_{\perp}^{2} /\left(2 u_{\beta \perp}^{2}\right)} \\
\times \int_{-\infty}^{\infty} d u_{\|} u_{\|} \frac{e^{-u_{\|}^{2} /\left(2 u_{\beta \|}^{2}\right)}}{z-n r_{\beta}-q_{\|} u_{\|}+i \tilde{\mathrm{v}}_{\beta}} \\
=\left(\frac{\tilde{\mathrm{v}}_{\beta}}{z}\right)^{2}(m !)(\sqrt{2})^{2 m+1}\left(1-\Delta_{\beta}\right) n_{\beta 0} u_{\beta \perp}^{2(m-1)} u_{\beta \|} \zeta_{\beta}^{0} \\
\times \frac{1}{\sqrt{\pi}} \int_{-\infty}^{\infty} d t \frac{t e^{-t^{2}}}{t-\hat{\zeta}_{\beta}^{n}}, \\
\times u_{\beta \perp}^{2(m-1)} u_{\beta \|} \zeta_{\beta}^{0}\left[1+\hat{\zeta}_{\beta}^{n} Z\left(\hat{\zeta}_{\beta}^{n}\right)\right] \\
J_{v v}\left(n, m ; f_{\beta 0}\right)=\left(\frac{\tilde{\mathrm{v}}_{\beta}}{z}\right)^{2}(m !)(\sqrt{2})^{2 m+1}\left(1-\Delta_{\beta}\right) n_{\beta 0}
\end{gathered}
$$

\section{Evaluation of the integrals $J_{v 0}\left(f_{\beta 0}\right)$}

We start from Eq. (15),

$$
J_{v 0}\left(f_{\beta 0}\right)=\int d^{3} u \frac{\tilde{v}_{\beta d}^{0}}{z} \frac{\mathcal{L}\left(f_{\beta 0}\right)}{u_{\perp}},
$$

Considering a bi-Maxwellian distribution, using Eq. (18), and using the average value of the collision frequency, one readily obtains

$$
\begin{gathered}
J_{v 0}\left(f_{\beta 0}\right)=-\frac{\tilde{v}_{\beta d}^{0}}{z} \frac{\left(1-\Delta_{\beta}\right) n_{\beta 0}}{(2 \pi)^{1 / 2} u_{\beta \perp}^{2} u_{\beta \|}} \\
\times \int_{0}^{\infty} d u_{\perp} e^{-u_{\perp}^{2} /\left(2 u_{\beta \perp}^{2}\right)} \int_{-\infty}^{\infty} d u_{\|} e^{-u_{\|}^{2} /\left(2 u_{\beta \|}^{2}\right)} \frac{u_{\|} u_{\perp}}{u_{\beta \perp}^{2}} .
\end{gathered}
$$

It is seen that the integral over $u_{\|}$vanishes in the case of a bi-Maxwellian distribution. Indeed, it vanishes for any distribution which is even in the parallel component of the velocity,

$$
J_{v 0}\left(f_{\beta 0}\right)=0 \text {. }
$$

\section{Evaluation of the integrals $J_{c h}\left(f_{\beta 0}\right)$}

The integral $J_{c h}$ is peculiar because it does not depend on the collision frequency. We start from Eq. (16),

$$
J_{c h}\left(f_{\beta 0}\right)=\int d^{3} u f_{\beta 0} \frac{1}{u} H\left(u^{2}+\frac{2 Z_{d 0} e q_{\beta}}{a m_{\beta} v_{*}^{2}}\right) .
$$

Considering a bi-Maxwellian distribution function, and using spherical coordinates, with $\mu=\cos \theta$, the integral can be written as follows,

$=\frac{n_{\beta 0}}{(2 \pi)^{1 / 2} u_{\beta \perp}^{2} u_{\beta \|}} \int_{-1}^{1} d \mu \int_{u_{\text {lim }}^{\beta}}^{\infty} d u u e^{-u^{2} /\left(2 u_{\beta \perp}^{2}\right)} e^{u^{2} \mu^{2}\left(1-\Delta_{\beta}\right) /\left(2 u_{\beta \perp}^{2}\right)}$,

where

$$
u_{\text {lim }}^{e}=\left(\frac{2 Z_{d 0}\left(e^{2} / a\right)}{m_{e} v_{*}^{2}}\right)^{1 / 2}, \quad u_{\text {lim }}^{i}=0 .
$$

Introducing a change of variables, $u^{2}=2 u_{\beta \|}^{2} t$, the integral is written as

$$
=\frac{n_{\beta 0}}{(2 \pi)^{1 / 2} u_{\beta \|} \Delta_{\beta}} \int_{-1}^{1} d \mu \int_{t_{l i m}^{\beta}}^{\infty} d t e^{-t\left[1+\mu^{2}\left(\Delta_{\beta}-1\right)\right] / \Delta_{\beta}},
$$

where

$$
t_{\text {lim }}^{i}=0, \quad t_{\text {lim }}^{e}=\left|\chi_{\|}^{e}\right|
$$

where we introduce the quantity $\chi_{\|}^{\beta}=q_{\beta}\left(Z_{d 0} e\right) /\left(a T_{\beta \|}\right)$, such that

$$
\chi_{\|}^{i}=\frac{Z_{d 0} Z e^{2}}{a T_{i \|}}, \quad \chi_{\|}^{e}=-\frac{Z_{d 0} e^{2}}{a T_{e \|}} .
$$

Performing the integration over the $t$ variable, and evaluating at the limits, we obtain

$$
=\frac{2 n_{\beta 0}}{(2 \pi)^{1 / 2} u_{\beta \|} \Delta_{\beta}} \int_{0}^{1} d \mu \frac{\Delta_{\beta}}{1+\mu^{2}\left(\Delta_{\beta}-1\right)} e^{-t_{\text {lim }}^{\beta}\left[1+\mu^{2}\left(\Delta_{\beta}-1\right)\right] / \Delta_{\beta}},
$$

where we have used the parity of the integrand on the $\mu$ variable.

The electron and ion contributions are therefore written as follows,

$$
\begin{gathered}
J_{c h}\left(f_{e 0}\right)=\frac{2 n_{e 0}}{(2 \pi)^{1 / 2} u_{e} \Delta_{e}} \int_{0}^{1} d \mu \frac{\Delta_{e}}{1+\mu^{2}\left(\Delta_{e}-1\right)} e^{-\left|\chi_{\|}^{e}\right|\left[1+\mu^{2}\left(\Delta_{e}-1\right)\right] / \Delta_{e}}, \\
J_{c h}\left(f_{i 0}\right)=\frac{2 n_{i 0}}{(2 \pi)^{1 / 2} u_{i \|} \Delta_{i}} \int_{0}^{1} d \mu \frac{\Delta_{i}}{1+\mu^{2}\left(\Delta_{i}-1\right)} .
\end{gathered}
$$

\section{APPENDIX B: EVALUATION OF THE AVERAGE VALUE OF THE COLLISION FREQUENCY, FOR A BI-MAXWELLIAN DISTRIBUTION}

The average value of the inelastic collision frequency is obtained by integration of the velocity dependent collision frequency over velocity space,

$$
v_{\beta}=\frac{1}{n_{\beta 0}} \int d^{3} u v_{\beta d}^{0}(u) f_{\beta 0}(u) .
$$


132

M.C. de Julie et al.

Using the expression for the collision frequency, which was introduced along with Eqs. (4) and (5), considering a biMaxwellian distribution, and using spherical coordinates, we obtain

$$
\begin{gathered}
\mathrm{v}_{\beta}=\frac{\pi a^{2} n_{d 0} v_{*}}{(2 \pi)^{1 / 2} u_{\beta \perp}^{2} u_{\beta \|}} \int_{0}^{\pi} d \theta \sin \theta \int_{0}^{\infty} d u e^{-u_{\perp}^{2} /\left(2 u_{\beta \perp}^{2}\right)} e^{-u_{\|}^{2} /\left(2 u_{\beta \|}^{2}\right)} \\
\times \frac{u^{2}}{u}\left(u^{2}-\frac{2 q_{d 0} q_{\beta}}{a m_{\beta} v_{*}^{2}}\right) H\left(u^{2}-\frac{2 q_{d 0} q_{\beta}}{a m_{\beta} v_{*}^{2}}\right) \\
=\frac{\pi}{\sqrt{2 \pi}} \frac{1}{u_{\beta \perp}^{2} u_{\beta \|}}\left(a^{2} n_{d 0} v_{*}\right) \int_{0}^{\pi} d \theta \sin \theta \\
\times \int_{u_{\text {lii }}^{\beta}}^{\infty} d u и e^{-u^{2} \sin ^{2} \theta /\left(2 u_{\beta \perp}^{2}\right)} e^{-u^{2} \cos ^{2} \theta /\left(2 u_{\beta \|}^{2}\right)}\left(u^{2}-\frac{2 q_{d 0} q_{\beta}}{a m_{\beta} v_{*}^{2}}\right) .
\end{gathered}
$$

Proceeding, with $\mu=\cos \theta$,

$$
\begin{gathered}
v_{\beta}=\frac{\pi}{\sqrt{2 \pi}} \frac{a^{2} n_{d 0} v_{*}}{u_{\beta \perp}^{2} u_{\beta \|}} \int_{-1}^{1} d \mu \\
\times \int_{u_{\text {lime }}^{\beta}}^{\infty} d u u e^{-u^{2} /\left(2 u_{\beta \perp}^{2}\right)} e^{u^{2} \mu^{2}\left(1-\Delta_{\beta}\right) /\left(2 u_{\beta \perp}^{2}\right)}\left(u^{2}-\frac{2 q_{d 0} q_{\beta}}{a m_{\beta} v_{*}^{2}}\right),
\end{gathered}
$$

with

$$
u_{\text {tim }}^{e}=\left(\frac{2 q_{d 0} q_{\beta} / a}{m_{e} v_{*}^{2}}\right)^{1 / 2}=\left(\frac{2 Z_{d 0}\left(e^{2} / a\right)}{m_{e} v_{*}^{2}}\right)^{1 / 2}, \quad u_{\text {tim }}^{i}=0 .
$$

Introducing a change of variables, $u^{2}=2 u_{\beta \|}^{2} t$, as in the calculation of (A16),

$$
\begin{gathered}
v_{\beta}=\frac{\pi}{\sqrt{2 \pi}} \frac{a^{2} n_{d 0} v_{*}}{u_{\beta \|} \Delta_{\beta}} \int_{-1}^{1} d \mu \\
\times \int_{t_{\text {lii }}^{\beta}}^{\infty} d t e^{-t / \Delta_{\beta}} e^{t \mu^{2}\left(1-\Delta_{\beta}\right) / \Delta_{\beta}}\left(2 u_{\beta \|}^{2} t+\frac{2 T_{\beta \|}}{m_{\beta} v_{*}^{2}} \chi_{\|}^{\beta}\right), \\
=(\sqrt{2 \pi}) \frac{u_{\beta} \|}{\Delta_{\beta}}\left(a^{2} n_{d 0} v_{*}\right) \int_{-1}^{1} d \mu \\
\times \int_{t_{\text {lime }}^{\beta}}^{\infty} d t e^{-t\left[1-\mu^{2}\left(1-\Delta_{\beta}\right)\right] / \Delta_{\beta}}\left(t+\chi_{\|}^{\beta}\right),
\end{gathered}
$$

where $t_{l i m}^{\beta}$ was defined along with Eq. (A16). This expression can be written as follows

$$
\begin{gathered}
v_{\beta}=2(\sqrt{2 \pi}) \frac{u_{\beta \|}}{\Delta_{\beta}}\left(a^{2} n_{d 0} v_{*}\right) \int_{0}^{1} d \mu \\
\times\left(-\frac{d}{d a} \int_{t_{l i m}^{\beta}}^{\infty} d t e^{-a t}+\chi_{\|}^{\beta} \int_{t_{l i m}^{\beta}}^{\infty} d t e^{-a t}\right),
\end{gathered}
$$

where $a=\left[1-\mu^{2}\left(1-\Delta_{\beta}\right)\right] / \Delta_{\beta}$. Performing the $t$ integrals,

$$
\begin{aligned}
& v_{\beta}=2(\sqrt{2 \pi}) \frac{u_{\beta \|}}{\Delta_{\beta}}\left(a^{2} n_{d 0} v_{*}\right) \int_{0}^{1} d \mu\left(\frac{d}{d a} \frac{e^{-a t}}{a}-\chi_{\|}^{\beta} \frac{e^{-a t}}{a}\right)_{t_{\text {lib }}^{\beta}}^{\infty} \\
& v_{\beta}=2(\sqrt{2 \pi}) \frac{u_{\beta} \|}{\Delta_{\beta}}\left(a^{2} n_{d 0} v_{*}\right) \int_{0}^{1} d \mu\left[\frac{e^{-a t}}{a}\left(-t-\frac{1}{a}-\chi_{\|}^{\beta}\right)\right]_{t_{l i m}^{\beta}}^{\infty}, \\
& v_{\beta}=2(\sqrt{2 \pi}) \frac{u_{\beta} \|}{\Delta_{\beta}}\left(a^{2} n_{d 0} v_{*}\right) \int_{0}^{1} d \mu \frac{\Delta_{\beta}}{1+\mu^{2}\left(\Delta_{\beta}-1\right)} \\
& \times e^{-t_{\text {lib }}^{\beta}\left[1+\mu^{2}\left(\Delta_{\beta}-1\right)\right] / \Delta_{\beta}}\left[t_{\text {nim }}^{\beta}+\frac{\Delta_{\beta}}{1+\mu^{2}\left(\Delta_{\beta}-1\right)}+\chi_{\|}^{\beta}\right] .
\end{aligned}
$$

For ions,

$$
\begin{gathered}
v_{i}=2(\sqrt{2 \pi})\left(a^{2} \varepsilon n_{i 0} v_{*}\right) \frac{u_{i \|}}{\Delta_{i}} \int_{0}^{1} d \mu \frac{\Delta_{i}}{1+\mu^{2}\left(\Delta_{i}-1\right)} \\
\times\left[\frac{\Delta_{i}}{1+\mu^{2}\left(\Delta_{i}-1\right)}+\chi_{\|}^{i}\right] .
\end{gathered}
$$

For electrons,

$$
v_{e}=2(\sqrt{2 \pi}) \frac{u_{e \|}}{\Delta_{e}}\left(a^{2} n_{d 0} v_{*}\right) \int_{0}^{1} d \mu \frac{\Delta_{e}}{1+\mu^{2}\left(\Delta_{e}-1\right)}
$$

$$
\begin{gathered}
\times e^{-\left|\chi_{\|}^{e}\right|\left[1+\mu^{2}\left(\Delta_{e}-1\right)\right] / \Delta_{e}}\left[\left|\chi_{\|}^{e}\right|+\frac{\Delta_{e}}{1+\mu^{2}\left(\Delta_{e}-1\right)}+\chi_{\|}^{e}\right] \\
v_{e}=2(\sqrt{2 \pi})\left(a^{2} \varepsilon n_{i 0} v_{*}\right) \frac{u_{e} \|}{\Delta_{e}} \int_{0}^{1} d \mu\left[\frac{\Delta_{e}}{1+\mu^{2}\left(\Delta_{e}-1\right)}\right]^{2} \\
\times e^{-\left|\chi_{\|}^{e}\right|\left[1+\mu^{2}\left(\Delta_{e}-1\right)\right] / \Delta_{e}} .
\end{gathered}
$$

Using the normalized variables,

$$
\tilde{\mathrm{v}}_{\beta}=\frac{\mathrm{v}_{\beta}}{\Omega_{*}}=2(\sqrt{2 \pi})\left(\varepsilon n_{i 0}\right) \frac{c^{3}}{\Omega_{*}^{3}} \frac{a^{2} \Omega_{*}^{2}}{c^{2}} \frac{\nu_{*}}{c} \frac{u_{\beta} \|}{\Delta_{\beta}} I^{\beta},
$$

where

$$
\begin{gathered}
I^{i}=\int_{0}^{1} d \mu \frac{\Delta_{i}}{1+\mu^{2}\left(\Delta_{i}-1\right)}\left[\frac{\Delta_{i}}{1+\mu^{2}\left(\Delta_{i}-1\right)}+\chi_{\|}^{i}\right], \\
I^{e}=\int_{0}^{1} d \mu\left[\frac{\Delta_{e}}{1+\mu^{2}\left(\Delta_{e}-1\right)}\right]^{2} e^{-\left|\chi_{\|}^{e}\right|\left[1+\mu^{2}\left(\Delta_{e}-1\right)\right] / \Delta_{e}} .
\end{gathered}
$$




\section{APPENDIX C: ANALYSIS OF THE EQUILIBRIUM CONDITION ON THE COLLISIONAL CHARGING OF THE DUST PARTICLES}

The condition of equilibrium for the collisional charging of the dust particles can be expressed simply as $\sum_{\beta} q_{\beta} n_{\beta 0} v_{\beta}=0$, which is equivalent to the expression using the normalized collision frequencies, $\sum_{\beta} q_{\beta} n_{\beta 0} \tilde{\mathrm{v}}_{\beta}=0$. Using the average collision frequencies, which are given by Eq. (B3), we can write the equilibrium condition as follows,

$$
\sum_{\beta} q_{\beta} n_{\beta 0} \frac{u_{\beta} \|}{\Delta_{\beta}} I^{\beta}=Z \frac{u_{i \|}}{\Delta_{i}} I^{i}-\left(Z-\varepsilon Z_{d 0}\right) \frac{u_{e} \|}{\Delta_{e}} I^{e}=0
$$

where we have used the ion charge $q_{i}=Z e$, and the neutrality condition to obtain the electron density,

$$
n_{e 0} e=n_{i 0} Z e-n_{d 0} Z_{d 0} e .
$$

Equation $(\mathrm{C} 1)$ can be solved to provide the value of the equilibrium charge of the dust particles, for a given value of the dust density $n_{d 0}=\varepsilon n_{i 0}$.

\section{ACKNOWLEDGMENTS}

M. C. de Juli was supported by the Brazilian Agency Fundação de Amparo à Pesquisa do Estado de São Paulo (FAPESP) (process number: 05/60160-5). L. F. Ziebell and R. Gaelzer acknowledge support from Conselho Nacional de Desenvolvimento Científico e Tecnológico (CNPq). The work was also supported by Fundação de Amparo à Pesquisa do Estado do Rio Grande do Sul (FAPERGS), Programa/Convênio PRONEX/CNPq.

This paper is gratefully dedicated to Dr. Ruth de Souza Schneider, whose unflagging enthusiasm and dedication to teaching and research inspired a whole generation of Physics students at Universidade Federal do Rio Grande do Sul. Dr. Schneider actively participated in the preparation of this manuscript almost up to her untimely passing away, at June $17,2008$.
[1] R. K. Varma, P. K. Shukla, and V. Krishan, Phys. Rev. E 47, 3612 (1993).

[2] M. R. Jana, A. Sen, and P. K. Kaw, Phys. Rev. E 48, 3930 (1993).

[3] V. N. Tsytovich and O. Havnes, Comm. Plasma Phys. Contr. Fus. 15, 267 (1993).

[4] S. I. Popel et al., Plasma Phys. Rep. 8, 284 (2004).

[5] J. R. Bhatt, Phys. Rev. E 55, 1166 (1997).

[6] N. D’Angelo, Planet. Space Sci. 42, 507 (1994).

[7] J. Vranjes, B. P. Pandey, and S. Poedts, Phys. Plasmas 9, 1464 (2002).

[8] J. J. Lee, J. Korean Phys. Soc. 43, 1587 (2004).

[9] L. F. Ziebell, R. S. Schneider, M. C. de Juli, and R. Gaelzer, Braz. J. Phys. 38, 297 (2008).

[10] G. E. Morfill, H. M. Thomas, U. Konopka, and M. Zuzic, Phys. Plasmas 6, 1769 (1999).

[11] J. E. Allen, Phys. Scripta 45, 497 (1992).

[12] V. N. Tsytovich, Sov. Phys. Usp. 40, 53 (1997).
[13] J. S. Chang and K. Spariosu, J. Phys. Soc. Japan 62, 97 (1993).

[14] V. N. Tsytovich, N. Sato, and G. E. Morfill, New J. Phys. 5, 43.1 (2003).

[15] M. C. de Juli and R. S. Schneider, J. Plasma Phys. 60, 243 (1998).

[16] M. C. de Juli and R. S. Schneider, J. Plasma Phys. 64, 57 (2000).

[17] V. N. Tsytovich, G. E. Morfill, and H. Thomas, Plasma Phys. Rep. 30, 816 (2004).

[18] B. P. Pandey and S. Kumar, Pramana - J. Phys. 45, 561 (1995).

[19] R. S. Schneider, L. F. Ziebell, M. C. de Juli, and V. JatencoPereira, Braz. J. Phys. 36, 759 (2006).

[20] M. C. de Juli, R. S. Schneider, D. A. Falceta-Gonçalves, and V. Jatenco-Pereira, IEEE Trans. Plasma Sci. 32, 542 (2004).

[21] M. C. de Juli, R. S. Schneider, L. F. Ziebell, and V. JatencoPereira, Phys. Plasmas 12, 052109 (2005). 